\title{
A corpus of 714 full-color images of depth-rotated objects
}

\author{
KARL VERFAILLIE and LUC BOUTSEN \\ Katholieke Universiteit Leuven, Leuven, Belgium
}

\begin{abstract}
A set of full-color images of objects is described for use in experiments investigating the effects of in-depth rotation on the identification of three-dimensional objects. The corpus contains up to 11 perspective views of 70 nameable objects. We also provide ratings of the "goodness" of each view, based on Thurstonian scaling of subjects' preferences in a paired-comparison experiment. An exploratory cluster analysis on the scaling solutions indicates that the amount of information available in a given view generally is the major determinant of the goodness of the view. For instance, objects with an elongated front-back axis tend to cluster together, and the front and back views of these objects, which do not reveal the object's major surfaces and features, are evaluated as the worst views.
\end{abstract}

A three-dimensional (3-D) object can project to an infinity of different two-dimensional (2-D) retinal images. However, under certain conditions, the visual system is able to acknowledge that two different images are projections of the same object, hence exhibiting viewpoint invariance or object constancy (Humphreys \& Quinlan, 1987; Verfaillie, 1992). For reasons of experimental control (e.g., precise control of the presentation time), most studies of object constancy do not use real 3-D objects as stimuli, ${ }^{1}$ but rather a 2-D portrayal of the object (e.g., a photograph or a line drawing). Once such a 2-D stimulus picture is available, it is relatively easy to produce an image of the same object in a different image-plane position (e.g., Biederman \& Cooper, 1991), size (e.g., Biederman \& Cooper, 1992) or image-plane orientation (e.g., Jolicoeur, 1985, 1988). It is much less obvious to produce stimulus representations of an object in different in-depth orientations. The reason is that the projected shape of an object is changed fundamentally after a rotation in depth: While image-plane rotations are isomorphic, indepth rotations are anisomorphic. Indeed, an in-depth rotation can result in a foreshortening of the object's major axis or the appearance and/or disappearance of object surfaces and features. For instance, the widely used stimulus set constructed and standardized by Snodgrass and Vanderwart (1980) cannot be employed to study effects of in-depth ro-

\footnotetext{
This research was supported by the Belgium Programme on Interuniversity Poles of Attraction, Convention No. 31. We are grateful to Jan Vaes, who created the object models (apart from models that were delivered with the 3D Studio software: according to the license agreement. images constructed on the basis of these models can be used unencumberedly), to Johan Van Rensbergen for technical advice, and to Gert Storms and two anonymous reviewers for comments on an earlier version of the manuscript. The full corpus of images in GIF format $(16 \mathrm{Mb})$ can be obtained from the FTP site at the Department of Psychology of the Katholieke Universiteit Leuven (ftp://michotte.psy.kuleuven.ac.be/pub/obj_db) or from the FTP site at Yale University (ftp://ftp.ctan.yale.edu/pub/obj_databank). We are grateful to Mike Tarr for providing this opportunity. Correspondence regarding the article should be addressed to Karl Verfaillie, Department of Psychology, Katholieke Universiteit Leuven, Tiensestraat 102. B3000 Leuven, Belgium (e-mail: karl.verfaillie( $\omega$ psy. kuleuven. ac.be).
}

tations, because the set contains only one perspective view per object. Therefore, most researchers of depth-rotation effects have constructed their own corpus of stimuli.

In a number of studies, subjects viewed pictures of unfamiliar objects in different in-depth orientations. Examples include brick objects used in mental rotation experiments (Metzler \& Shepard, 1974), Bülthoff and Edelman's (1992; Edelman \& Bülthoff, 1992), wire-frame and amoebalike objects, and the arbitrary machined tool part used by Langdon, Mayhew, and Frisby, 1991.

Some experiments have focused on the perception of a restricted class of real-world, nameable objects shown in different in-depth orientations. The stimuli are mostly biological objects or object parts, such as faces (e.g., Bruce, Valentine, \& Baddeley, 1987; De Renzi, Scotti, \& Spinnler, 1969; Roberts \& Bruce, 1989), hands and feet (Parsons, 1987b), or whole bodies shown by means of photographs of humanlike models (Parsons, 1987a) or under point-light conditions (Verfaillie, 1993; Verfaillie, De Troy, \& Van Rensbergen, 1994).

Other studies have used a larger class of real-world objects. Most of the corpi consist of either line drawings or photographs, sometimes digitized to present on a computer screen. Table 1 lists a sample of studies, including the number of objects tested, the number of exemplars of each object, and the number of views of each exemplar. These numbers do not include the number of objects used for practice or demonstration, the objects for which only one view was constructed, or the number of available imageplane rotated versions. There is a tradeoff between the number of objects and the number of views available. Palmer, Rosch, and Chase (1981) used a large number of views (12) but employed only 12 objects. The corpus of Biederman and Gerhardstein (1993) contains more objects (two instances of 24 objects), but there are only three views of each object.

In this article, we describe a new corpus containing a large number of objects (70). Moreover, up to 11 views per object are available. For some objects, the number of dif- 
Table 1

Number of Objects, Exemplars of Each Object, and Views of Each Exemplar Used in Studies on the Effect of Depth-Rotation on Object Recognition

\begin{tabular}{lcccr}
\hline \multicolumn{1}{c}{ Study } & Objects & Exemplars & Views & Total \\
\hline Photographs & & & & \\
Bartram, 1976, Experiment 2 & 10 & 1 & 8 & 90 \\
Ellis \& Allport, 1986 & 20 & 2 & 2 & 80 \\
Ellis, Allport, Humphreys, & 20 & 2 & 2 & 80 \\
$\quad$ \& Collis, 1989 & & & & \\
Humphreys \& Riddoch, 1984 & 47 & 1 & 3 & 141 \\
Kelter et al., 1984, Experiment 1 & 18 & 1 & 2 & 36 \\
Layman \& Green, 1988 & 30 & 1 & 2 & 60 \\
Marshall \& Walker, 1987 & 2 & 2 & 2 & 8 \\
Palmer et al., 1981 & 12 & 1 & 12 & 144 \\
Srinivas, 1993, & & & & \\
$\quad$ Experiments 3 and 4 & 42 & 1 & 2 & 84 \\
$\quad$ Warrington \& Taylor, 1973 & 20 & 1 & 2 & 40 \\
Line drawings & & & & \\
Bartram, 1976, Experiment 1 & 8 & 2 & 2 & 32 \\
Biederman \& Gerhardstein, 1993 & 24 & 2 & 3 & 144 \\
Humphrey \& Jolicoeur, 1988 & 32 & 1 & 2 & 64 \\
Kelter et al., 1984, Experiment 2 & 15 & 1 & 2 & 30 \\
\hline
\end{tabular}

ferent views is smaller. This is the case when the object contains several planes of symmetry, so that it projects to the same image when seen from different vantage points (in the extreme case of a uniform sphere, all views would be equivalent). The total number of images amounts to 714 . In contrast to stimulus sets containing line drawings or blackand-white photographs, all images of the present corpus are full color. Black-and-white, low-resolution renderings of the images can be found in Appendix A. Figure 1 depicts a sample $640 \times 480$ pixel image printed with a resolution of 300 DPI. The image in Figure 1 and some additional example images are accessible through World Wide Web (http://www.psy.kuleuven.ac.be/labexp/labexp.html).

The complete set of images is available as GIF (Graphics Interchange Format) files (see authors' note for more details on the availability). This has the advantage that they can be transformed relatively easily. For instance, several software packages allow the gray-scaling of color GIF images. Procedures for Euclidean transformations of GIF files, including scaling and image-plane translations and rotations, are also readily available.

Apart from a technical description of the corpus, this article also provides ratings of the degree to which each 2-D view affords recognition of the 3-D object. More specifically, in a paired-comparison experiment, subjects selected the "best" view in each pair of views for each object. Thurstonian scaling solutions of subjects' preferences were constructed, resulting in a scale of "goodness of view" for each object (Appendix A). ${ }^{2}$ This will make it possible to use the corpus in future studies of the influence of goodness of view on specific measures of recognition (e.g., Humphreys \& Riddoch, 1984, 1985; Srinivas, 1993).

Finally, we performed an exploratory cluster analysis on the scaling solutions. This allowed us to examine whether different classes of objects can be discerned depending on the best (and/or worst) views and what factors determine the goodness of a view. To anticipate the results, we ob- served that cluster membership was primarily governed by the coarse physical structure of the object. For instance, objects with an elongated front-back axis tended to cluster together and the front and back views of these objects (in which the observer's line of sight is parallel to the object's major axis) were evaluated as the worst views. We would like to stress the exploratory nature of this cluster analysis. Future rating studies (e.g., in which subjects judge the saliency of particular features or axes in each view) should shed more light on the precise characteristics determining view preferences.

\section{METHOD}

\section{Stimuli}

Selection of the objects. The corpus contains 70 different objects. We made no attempt to construct a "representative" set, such as the set of Snodgrass and Vanderwart (1980). One of the criteria that Snodgrass and Vanderwart used in selecting their objects was that the set included exemplars from well-studied categories (Battig \& Montague, 1969). Except for four animals, all objects in the present corpus are man-made objects. To be representative, the set should have included more animals, in addition to fruit and vegetables and parts of the human body. However, most studies employing Snodgrass and Vanderwart's stimuli use only a subset of the corpus, and there is rarely an attempt to preserve the representativeness of the overall corpus in the subset. Moreover, one reason for the preponderance of man-made objects in our set is technical: Natural objects predominantly contain smooth surfaces, and these are difficult to handle in the software package that we used to produce the images.

Selection of the orientations. To construct the images of different views, each object was placed in an imaginary viewing sphere. Figure 2 shows the duck placed in the viewing sphere. The duck has an intrinsic orientation (Carlson-Radvansky \& Irwin, 1993; Garnham, 1989; Levelt, 1984) - that is, it has an evident top-bottom and front-back axis (and therefore also a left-right axis). Moreover, the object is mono-oriented-that is, it normally appears in an upright orientation in an environment-centered reference frame. For this type of object, the object's position and orientation in the viewing

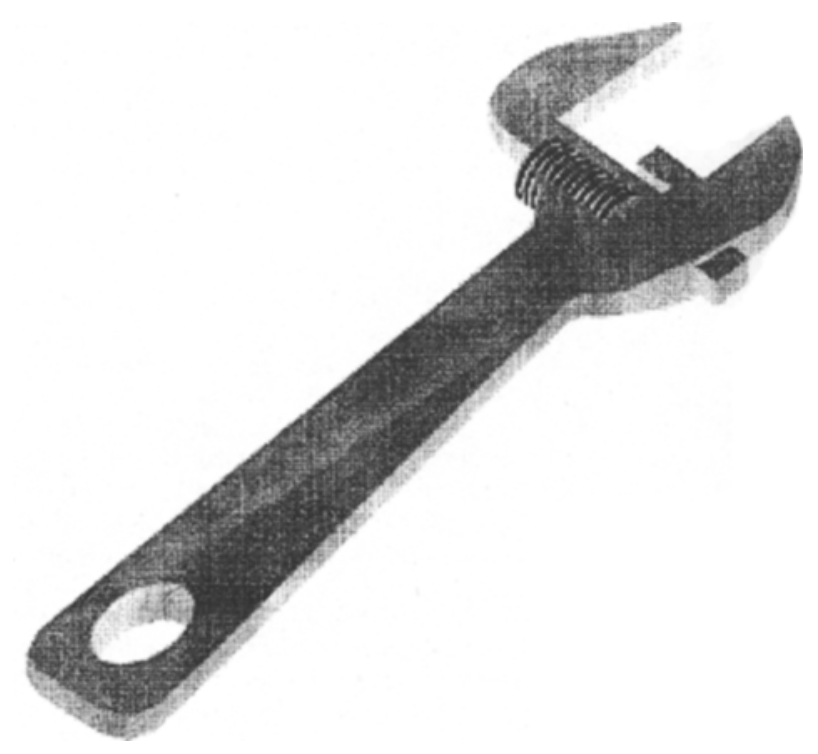

Figure 1. A sample image (printed with 300 DPI) showing a 3/4 view of a spanner observed from an elevated viewing angle. 


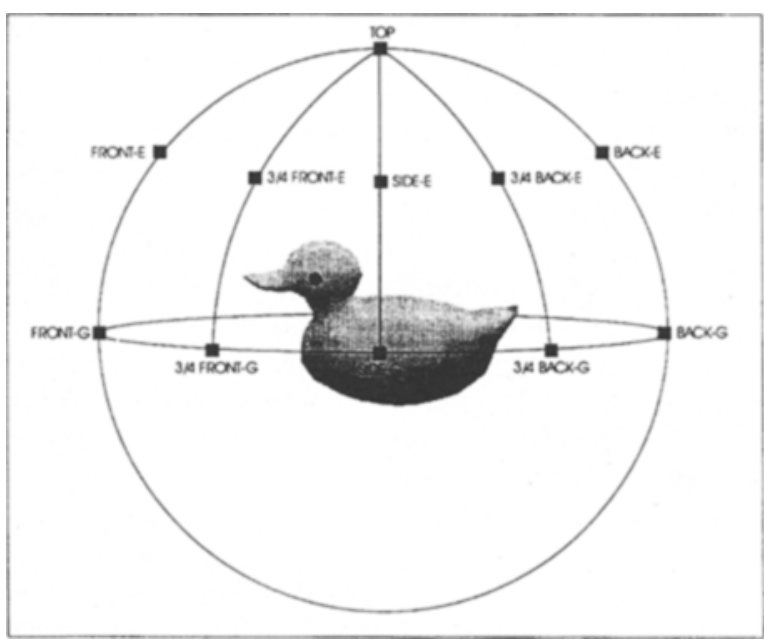

Figure 2. A duck placed in the viewing sphere and the 11 perspective views.

sphere guarantees that its top-bottom axis, front-back axis, and left-right axis correspond with the sphere's $y$-axis (the vertical axis through the sphere's center in Figure 2), z-axis (the horizontal axis through the sphere's center in Figure 2), and $x$-axis (the axis orthogonal to the other two axes), respectively. When the point where these three axes intersect coincides with the object's center of gravity, the three axes define three planes running through the object. The midsagittal (or median) plane contains the $y$-and $=$-axes, the midfrontal (or coronal) plane contains the $x$-and $y$-axes, and the midtransterse plane contains the $x$ - and $z$-axes (Howard. 1982).

For some poly-oriented objects (objects that lack a prototypical upright orientation, such as a knife), one can sometimes discern a top-bottom, a front-back, and a left-right axis. However, there is much less consensus among raters. We adopted the following strategy to locate these objects in the viewing sphere. Most poly-oriented objeets in our set are utensils. The axis of elongation was aligned with the sphere's $z$-axis. The handle was located on the positive side of the axis, which defines the front of the object (for objects that lack a distinct handle, such as the pen, pencil, and corkscrew, the "most important" object part [e.g., the point of the pen] was located on the positive side of the $=$-axis). In addition to an axis of elongation. most objects in the present set also are (approximately) symmetrical. In fact. most objects contain several planes of symmetry. The object was placed in the viewing sphere in such a way that its main plane of symmetry coincided with the midsagittal plane in the viewing sphere. This strategy capitalized on the fact that most mono-oriented objects in our corpus also are symmetrical across the midsagittal plane.

Figure 2 depicts the 11 selected views. Palmer et al. (1981) used the same 11 views (in addition to a 12 th view taken from a vantage point that reflected the authors' intuitions about the object's most canonical orientation). There are 5 views in which the observer's line of sight is orthogonal to the equator of the viewing sphere. In Front$G$ ( $G$ stands for ground level to indicate that the object is depicted from a nonelevated viewing angle) and Back-G, the observer's line of sight coincides with the two ends of the $z$-axis. In Side-G, the object is shown in a lateral view. In the $3 / 4$ Front-G and $3 / 4$ Back-G views, the object is in an orientation between the Front-G and Side$G$ views and the Back- $G$ and Side- $G$ views, respectively. Corresponding to these 5 views, there are 5 orientations in which the object is shown from an elevated viewing angle: Front $E$ ( $E$ stands for clevated), Side-E, Back-E, 3/4 Front-E, and 3/4 Back-E. The final view shows the top of the object.
For 13 of the 70 objects, 2 or more of the 11 views are identical, because the objects contain several planes of symmetry. ${ }^{3}$ In total, the corpus contains 714 different images.

In all views except the top view, the top-bottom $y$-axis appears vertical in the image. Because the $y$-axis is oriented in the direction of gravity, this means that mono-oriented objects are always shown in an upright orientation. For the top view (in which the $y$-axis coincides with the observer's line of sight), the front-back axis appears horizontal in the image and the object is oriented to the left. This is also the case in Palmer et al.'s study.

The orientations on the left side of the viewing sphere were not selected. Because almost all objects in the present corpus are (approximately) symmetrical across the midsagittal plane (the piano and the telephone are the least symmetrical objects), views taken from a vantage point on the left side of the sphere (e.g., the rightward sagittal view) and views taken from a point on the right side (e.g., the leftward sagittal view) are (approximately) mirror reflections of each other. ${ }^{4}$

Construction of the images. Images were constructed with $\mathrm{Au}$ todesk 3D Studio (Autodesk, Inc., 1993), a 3-D modeling and animation application program. The package allows the user to build a 3-D model of an object and apply diverse materials (defined by colors, transparency, texture, reflection, etc.) to selected parts of the model. The 3-D model can then be placed in a 3-D environment, and different lights and spotlights can be added to the scene. By rotating the object in depth, images of the object viewed from different vantage points are made. The program also makes it possible to save the image in GIF format (among other possible formats).

After constructing the 3-D models and adding materials, the model of each object was scaled until all objects had exactly the same size along their longest axis. This implies that the 70 objects were matched as far as their projected size is concerned in the image with the largest extent (e.g., the side views of the truck). In their largest horizontal extent, the objects occupied almost half of the screen width $(49 \%)$

The 3-D object model was illuminated by four lights. The spots were placed in four opposite corners above the object model and were directed toward the center of the object. When necessary, four lights were added at the level of the cquator of the viewing sphere. The falloff (the outer extremity of the circle of light cast by the spot, i.c., the circumference of the circle where the pool of light meets darkness) was defined as $90^{\circ}$. The hotspot (the bright circle in the center of the pool of light cast by the spot, i.e., the circumference of the circle within which intensity is highest and constant) was defined as $45^{\prime \prime}$. In the case of overexposure, light intensity was adjusted. There were no cast shadows in the image.

Objects wcre presented on a uniform white background without texture. Therefore, the background contained no depth cues. This is important, because Humphrey and Jolicoeur (1993) showed that the addition of a background with monocular depth cues can improve identification of objects depicted in unconventional in-depth orientations.

\section{Procedure}

Sixty-three students ( 35 women and 28 men) of the Katholieke Universiteit Leuven participated in the experiment. All had normal or corrected-to-normal vision. They were divided into seven groups of 9 subjects. Each subject was tested individually. The experiment took about $1.5 \mathrm{~h}$.

The 70 objects were arranged in seven sets of 10 objects. Each set contained an approximately equal number of mono-oriented and poly-oriented objects. The objects for which less than 11 different views were available were also divided across the seven sets. Finally, care was taken that objects from a similar class (e.g., vehicles) did not all occur within the same set.

Each group of subjects received one set of objects. For each object, all $\left(\begin{array}{l}1 \\ 2\end{array}\right)$ pairs of views were administered. In each pair of views, the 
subjects judged in which view the object was most easily recognized. Presentation order of the objects within a set, of the object pairs for a particular object, and of the two views within each pair of views was randomized. The subjects judged all pairs of views of a particular object before the next object was administered.

Stimuli were presented on a 17 -in. super VGA color monitor. The images were displayed with a resolution of $640 \times 480$ pixels. The time course of a trial was as follows. First, the name of the object appeared on the screen (because it was possible that the first pair of views consisted of two views that were hard to identify). After the subject pressed a button, the first view of the pair appeared. With another buttonpress, the first orientation disappeared and the second view was administered. The subject could toggle between the two views until one of the orientations was selected as the best view. The screen was cleared, and the subject received the next pair of views. The subjects could take a break between two objects.

The subjects received detailed written instructions. It was explained that objects generally can be viewed from different vantage points and that some perspective views do allow an observer to recognize the object more easily than do other views. The experimenter demonstrated the paired-comparison task with an object (a mixer) that did not occur in the experimental stimulus set. The subject performed two training trials with pairs of views of this object.

\section{Results and Discussion}

Scaling. To determine the degree of consistency within subjects, the number of circular triads was ascertained for each subject's judgment of each object. A circular triad occurs when a subject is intransitive in his/her preference for three views: View $i$ is preferred over view $j$ and view $j$ is preferred over view $k$, but view $k$ is preferred over view $i$. The second column of Appendix B lists the median number of circular triads for each object, ranging from 0 to 12. Kendall (1962) developed a coefficient of consistency, $\zeta$, that takes into account the number of observed circular triads and the maximum number of possible circular triads. Kendall also discussed a method to test the significance of $\zeta$. For each subject's judgment of each object ( 9 subjects $\times 70$ objects), the $\chi^{2}$ value was significant. This indicates that, in all judgments, there was a larger degree of consistency than would be expected if the subjects had made their judgments at random.

On the basis of Kendall's (1962) coefficient of agree$m e n t, u$, the degree of consistency across subjects was determined. The $u$ values and the $\chi^{2}$ value used to test the significance of $u(d f \mathrm{~s}=81,53,31,15$, and 4 for 11 -view, 9-view, 7-view, 5-view, and 3-view objects, respectively) can be found in the third and fourth columns of Appendix B, respectively. The $\chi^{2}$ value was always significant ( $p$ s $<.001$, except for the saucer, where $p<.05$ ), indicating that the probability that the subjects had reached the level of agreement by chance was low.

We decided that the degree of consistency was sufficiently high to construct Thurstonian Case V scaling solutions of subjects' preferences among the views (Thurstone, 1927; Torgerson 1958). The scaling solutions are part of the figures in Appendix A. The best view is given the zero point of the scale. This point is arbitrary, since we are dealing with an interval scale. The unit of scaling is (also arbitrarily) equal to one standard normal deviate.

When all subjects agree that view $j$ is better than view $k$ (the next best view), the proportion of times that view $j$ is preferred over view $k$ is 1.00 . When all subjects agree that view $j$ is worse than view $i$ (the preceding best view), the proportion of times that view $j$ is preferred over view $i$ is 0.00 . The normal distribution being asymptotic, the unit normal deviates corresponding to these proportions (the $z$-score) cannot be obtained (and this is a necessary step in the construction of a Thurstonian scaling solution). Some authors have proposed replacing proportions of 1 and 0 by slightly smaller and greater values, respectively. For instance, Dunn-Ranking (1983) reduces proportions greater than .98 to .98 and increases proportions smaller than .02 to .02. This would allow one to obtain a $z$-score for these proportions after all. However, the $z$-scores associated with these extreme proportions are still unreliable. Guilford (1954, p. 163) cautioned against using $z$-values more extreme than +2.0 and -2.0 (corresponding to proportions of .977 and .023 , respectively). Therefore, in the computation of the scaling values, we omitted the extreme values from the $z$-matrix. Normally, this poses no problem, because several estimates of the separation between successive stimuli are available. Torgerson (1958) describes this procedure for finding a scaling solution on the basis of incomplete $z$-matrices in more detail.

However, in some cases, there were no available estimates of the separation between a view and the immediately preceding and/or following view on the interval scale. This occurs when all subjects consider the best view better than all other views or the worst view worse than all other views. It also occurs when all subjects agree that an intermediate view $j$ is worse than all better views and the immediately preceding view $i$ is better than all worse views. In this case, it is impossible to compute the separation between views $i$ and $j$. This is not problematic: it simply indicates that views $i$ and $j$ are far apart on the scale (or at least much farther apart than other pairs of views).

However, to make comparisons across objects, we thought that it may be interesting to have a scale value for each view of each object. Therefore, for those pairs of views for which the separation on the interval scale was not estimable, we arbitrarily assumed a separation of two standard normal deviates, which is equivalent to the case in which $98 \%$ of the subjects prefer view $i$ over view $j$. The views, arbitrarily separated by two normal deviates from the preceding view, are marked with a small asterisk in the figures in Appendix A. We had to adopt this strategy for 21 of the 7511 -view objects, 4 of the 79 -view objects, the only 7-view object, and 3 of the 43 -view objects.

To test the goodness of fit of the scaling solutions, expected proportions were obtained from the estimated scale values. The results of Mosteller's (1951) test comparing the inverse-sine transformations of observed and expected proportions are shown in the fifth column of Appendix B. The $\chi^{2}$ value is frequently significant, indicating that the data are not fully accounted for by the model. The relatively high proportion of significant values is not due to our strategy of assuming an arbitrary separation of two standard normal deviates when a particular interval was not estimable. The fit is not worse for the objects for which we 
adopted this strategy than for the other objects. In fact, the trend is the opposite.

Some authors have cautioned against the use of Mosteller's (1951) test because it underestimates the fit (e.g., Torgerson, 1958, pp. 187-188). Gulliksen and Tukey's (1958) coefficient of reliability, which expresses the proportion of explained variance, does not have this disadvantage. The values are given in the sixth column of Appendix $\mathrm{B}$. These measures of goodness of fit can be taken into account in future research on the effect of goodness of view on recognition.

Clustering. To examine what factors might determine the goodness of a view, the 11-view objects were hierarchically clustered on the basis of their scale value for each view. Clustering was accomplished by means of the CLUSTER procedure of the SAS statistical package (SAS Institute, Inc., 1990). We selected the equal-variancemaximum-likelihood method (SAS, 1990, pp. 533-534) from the available clustering methods. In most simulation studies with clusters of roughly equal size, Ward's minimumvariance method is evaluated best (SAS, 1990, p. 56). The equal-variance-maximum-likelihood method is similar to Ward's method, but it removes the bias of the latter method toward clusters of equal size. Procedures for estimating the number of clusters suggested that there are 8 clusters (Milligan \& Cooper, 1985; SAS, 1990, pp. 97-99): The pseudo $F$ statistic was highest at 8 clusters (except for a local peak at 2 and 3 clusters), and pseudo $t^{2}$ was small at 8 clusters and large for the next cluster fusion.
Figure 3 displays a tree diagram of the cluster hierarchy. The objects in Figure 3 (as well as in Appendix A) are ordered as a function of the degree of membership within their cluster. The degree of membership of an object was determined by the sum (over the scale values) of the squared difference between the object's scale value and the mean scale value of all objects within the cluster.

The clusters can be characterized by the coarse physical structure of their member objects and the way this structure is oriented in the viewing sphere. More specifically, the degree to which the object is spatially extended in the direction of the $x-y$-, and $z$-axes determines the goodness of specific views and therefore also cluster membership.

The 1 st cluster contains 10 objects, with the roller skate as its most typical member. The objects mostly have evident top-bottom and front-back axes (except for the razor and the pitchfork). The physical extension in the direction of the front-back axis is most pronounced, giving the objects an elongated appearance. However, the objects are not flat (this distinguishes this cluster from the 4th and 5 th clusters); instead, they are also extended along the other two axes, although the extension along the left-right axis tends to be slightly more prominent than along the topbottom axis. Given the elongation along the front-back axis, the foreshortened views that reveal only the object's smallest surfaces (i.e., Front-G and Back-G, revealing the object's front and back, respectively) are generally considered to be the worst views. The views simultaneously

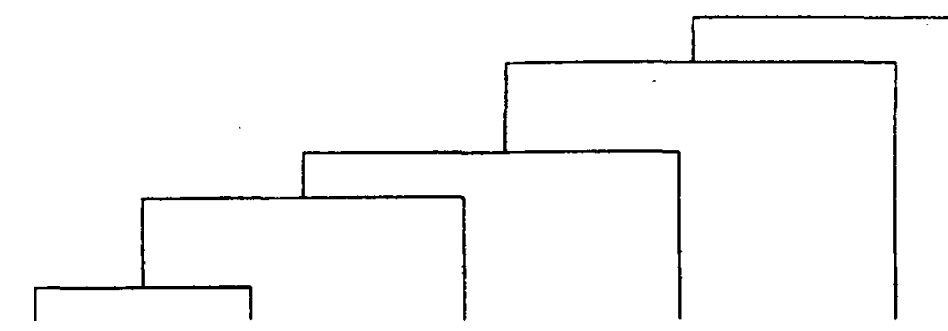

1

roller skate
piano
stapler

skateboard

tricycle

razor blade

fighter jet

shoe

pitchfork

pan
2

ventilator
extinguisher
globe

concrete mixer

desk lamp

perforator

call box

watch

oil lamp
3

cow
helicopter shaver
car
truck
train
roller
chicken
duck
teapot
fork-lift
bench

8
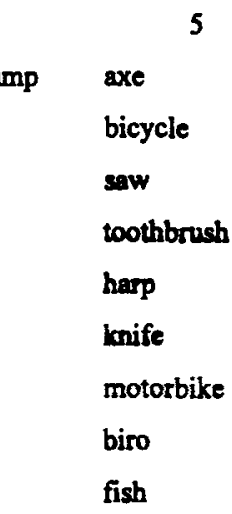

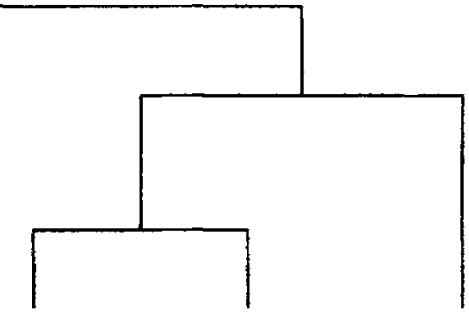

4

6

7

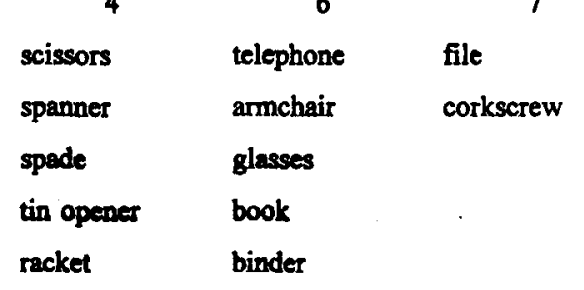

Figure 3. The cluster tree of the 11-view objects. 
uncovering the front, side, and top ( $3 / 4$ Front-E) or the back, side, and top (3/4 Back-E) are evaluated as the best views.

The ventilator is the prototypical member of the 2 nd cluster, which includes 9 objects. As in the 1st cluster, all objects have an obvious upright orientation. The objects are also extended in all three dimensions, but, in contrast to the 1st cluster, the extension in the direction of the front-back axis is the least pronounced. Most objects are more or less elongated along their top-bottom axis. Therefore, the view that reveals only the object's top is the worst view (except for the concrete mixer, where the top is the last but one view on the scale). The $3 / 4$ views are again the best views. Note that the $3 / 4 \mathrm{G}$ views (which do not reveal the top) sometimes precede the $3 / 4 \mathrm{E}$ views (which also show the top) on the scale. This can be explained by the fact that the top view reveals only the smallest surface of the object, and, therefore, the perceptibility of the top in the $3 / 4 \mathrm{E}$ views does not make them much better than the $3 / 4$ $G$ views.

The 3 rd cluster contains 11 objects, mostly vehicles and animals. The cow is the most prototypical member. Parallel with the 1 st cluster but in contrast to the 2 nd cluster, the objects are elongated along their front-back axis (except for the bench, which is the most atypical member). In contrast to the 1 st cluster, the top plane is not judged to be important. First, for some objects, the second most pronounced elongation occurs along the top-bottom axis: The side planes are more extended than the top plane. Second, for some objects this is not the case: The extension of the side is approximately equal to the extension of the top plane, as in the truck, or is even less pronounced than the extension of the top plane, as in the car. However, these objects normally appear upright. Moreover, they are relatively large, relative to humans. Therefore, they rarely reveal their top to an upright human observer, making the top plane less diagnostic for the object's identity than the side plane. The importance of the side and, to a lesser degree, the front explains the fact that the 3/4 Front-G view is rated as the best view for all objects (except for the teapot, where it is judged as the second best view). The observation that the $3 / 4$ Front-G view is preferred over the $3 / 4$ Front-E view, although the latter view reveals the top in addition to the side and the front, can be related to the insignificance of the top. The importance of the side can also explain the observation that the 5 worst views of all objects are the only 5 views that hide the side, and the 6 best views all reveal the side (except for the roller, where Front-G and Side-G are switched):

The scissors represent the prototypical member of the 4th cluster, which incorporates 9 objects. All objects (except the rubber dinghy) are poly-oriented - that is, they have no conventional upright orientation. The objects are elongated along the $z$-axis. Moreover, and this sets them apart from the objects of the preceding 3 clusters, the objects in the 4th cluster are more or less flat in the midtransverse plane: The objects are essentially 2-D. The elongation along the $z$-axis explains the observation that the Front- $G$ and Back- $G$ views are evaluated as the worst views for 7 of the 9 objects. In these views, the objects are extremely foreshortened. Because the objects are flat, the other $3 \mathrm{G}$ views, which reveal the side of the object (in addition to the front or the back), still do not uncover the crucial top plane. Therefore, the Side-G, 3/4 Front-G, and 3/4 Back-G views do not provide much additional information, relative to that provided by the foreshortened Front-G and Back-G views. Consequently, for 6 of the 9 objects, the 5 worst views are the $5 \mathrm{G}$ views. Conversely, the 6 best views generally reveal the top of the object.

The 5th cluster includes 9 objects, mono-oriented (e.g., the bicycle) and poly-oriented (e.g., the axe). The axe is the most prototypical exemplar. Parallel with the 4th cluster, the objects are elongated along the $z$-axis and they are essentially 2-D. In contrast to the fourth cluster, they are not flat in the midtransverse plane but in the midsagittal plane. ${ }^{5}$ Because of the elongation along the $z$-axis, the objects are foreshortened in the Front- $G$ and Back-G views. The latter 2 views are rated as the worst views for all objects (except for the motorbike). Parallel to the 4th cluster, which also contains 2-D objects, one plane plays a privileged role in determining goodness of view. Whereas the top is crucial in the 4th cluster, the side plane is critical in the 5 th cluster. For all objects (except for the toothbrush, where Front-E and 3/4 Back-G views are switched), the 5 views that conceal the side are considered as the 5 worst views. Conversely, the 6 views showing the side are evaluated as the 6 best views. In fact, for 6 of the 9 objects, all subjects agree that the 6 views that uncover the side are better than the 5 views that conceal the side, so that the two groups of views are arbitrarily separated by two standard normal deviates.

The most prototypical member of the 6th cluster, which includes 5 objects, is the telephone. At first sight, this cluster is the most heterogeneous cluster in terms of the structural correspondence between its member objects. Some objects, such as the telephone, are more or less flat in the midtransverse plane. Other objects, such as the book, are relatively flat in the midsagittal plane. One object, the armchair, is equally extended in all three directions. What seems to unify the objects is the fact that their main sides are straight and that these sides are parallel with one of the axes of the viewing sphere. This causes, especially in the range of views selected in this study, "accidents" of viewpoint: Regular 2-D shapes, such as disks and rectangles that are not diagnostic of the 3-D object, emerge in the image. The glasses are a good example: The 2 worst views depict two circles connected by straight lines, a highly regular pattern that does not correspond to the 3-D configuration. It is not surprising, then, that the better views are those views in which the observer's line of sight is not orthogonal to at least two of the three main planes. This is the case for the $3 / 4$ views, where the line of sight is not parallel with the midsagittal and the midfrontal plane, and for the E views, where the line of sight is not parallel with the midtransverse plane and the midsagittal or the midfrontal plane. For most objects, the $3 / 4$ Front-E view is considered to be the best view (except for the glasses, where the $3 / 4$ Front-E view is the second best view and the $3 / 4$ Back-E view is the best view). In this orientation, the line of sight is 
nonorthogonal to all three planes. Conversely, the Front-G, Back-G, Side-G, and Top views, where the line of sight is orthogonal to two of the three main axes (and therefore parallel with the third axis), tend to be the worst views.

The final 2 clusters each contain only two objects. The 7 th cluster includes the file and the corkscrew. Relative to the other clusters, the scale values have a large dispersion. The file and the corkscrew are the only objects with two scale values larger than six standard normal deviates. This is partly due to the high consistency among subjects. In the case of the corkscrew, there are 3 pairs of views for which all subjects agree that the worse view of the pair is worse than all better views and the better view is better than all worse views. The 2 views of such a pair are arbitrarily separated by two standard normal deviates, which increases the variability of the scale values. For both objects, the Front-G and Back-G views are rated as the worst views. Because the objects are elongated along the $z$-axis, foreshortening is extreme in these 2 views. The objects are relatively flat in the midtransverse plane, so that the top is more informative than the sides. Therefore, the $5 \mathrm{G}$ views (which conceal the top) are the 5 worst views. The $3 / 4$ Front-E and 3/4 Back-E views, which show object parts in the three main planes, are evaluated as best.

The petrol pump and the shaver are the only 2 objects in the 8th cluster. The objects are relatively elongated along the top-bottom axis. This makes the top the worst view. On the other hand the objects are not flat in one of the two other dimensions. Moreover, due to functional properties of the objects, the front plays a more important role than the back. The views containing the nondiagnostic back side are generally considered less prototypical than the views containing the front side.

\section{GENERAL DISCUSSION}

According to Palmer et al. (1981), the goodness of a view is controlled by two factors: the objectively available information and the salience of this information for the viewer. Both factors determine the "subjective information content" of a given view, which is maximized in the best view.

The goodness-of-view ratings in the present study corroborate the importance of the first factor. Since most realworld objects are opaque, only part of the entire 3-D object is visible from a particular vantage point. Therefore, the orientation of a specific object in the viewing sphere governs the amount of information available in a given view, and this is the principal determinant of the goodness of the view. For the majority of the objects in the present set, the $3 / 4 \mathrm{E}$ views are rated as the best views. These views simultaneously reveal aspects about the object's side, top, and front or back. The views disclosing only two of the three major planes generally are considered less good. When the observer's line of sight is parallel with one of the three main axes of the viewing sphere, only one plane (the front, back, side, or top) is visible. In general, these views are evaluated as worse. Moreover, the view that reveals only the smallest surface is generally the worst view.
For instance, in the 1st cluster, which contains objects that are elongated along the front-back axis, the front and back are rated as the worst views, whereas, in the second cluster, which contains objects that are elongated along the top bottom axis, the top is the worst view. These views not only obscure most of the object's parts by foreshortening the object's main axis they also make it difficult to recover the spatial arrangement of the parts, a prerequisite for recognition according to some theories of object perception (e.g., Marr \& Nishihara, 1978). Finally, bad views may sometimes "mislead" the visual system, because the proximal 2-D information does not adequately specify the 3-D distal structure (see 6th cluster).

Whereas the first factor has to do with the objectively available information, the second factor relates to the subjective salience of this information for the identity of the object. According to Palmer et al. (1981), it pertains to "the perceiver's familiarity with the revealed aspects and their importance within his/her knowledge about objects" (p. 147). Although the first factor turns out to be more crucial, there are some indications in our data that the second factor also plays a role. First, the views containing the front surface are sometimes judged to be better than the corresponding views, including the back surface, even though the front does not necessarily reveal more information objectively than does the back. For some objects, this can be linked to the fact that they have a typical direction of motion ( such as the objects in the 3 rd cluster, which mainly consists of vehicles and animals). For other objects (such as the piano, the concrete mixer, and the bench), the front is more important during physical interaction between the observer and the object. Second, for some mono-oriented, relatively large objects that seldomly reveal their top surface to a comparatively smaller observer, the views revealing the top surface (the E views) are not considered as more canonical than the views hiding the top surface (the $\mathrm{G}$ views).

In general, the better views not only maximize the subjective information content (Palmer et al., 1981) they also tend to be the most stable views. In a stable view, the object may be rotated (or the observer may perform exploratory movements) without affecting the qualitative structure of the image (Koenderink \& van Doorn, 1976, 1979; also, Koenderink \& van Doorn, 1977, provide a nontechnical introduction) - that is, the same surfaces remain visible. Because most objects in the present corpus have straight surfaces and these surfaces frequently parallel one of the three axes of the viewing sphere, the most stable views are those views where the observer's line of sight is not parallel with one of the three axes of the viewing sphere (the 3/4 Front-E and 3/4 Back-E views). In an unstable view, only a small rotation of the object is sufficient for the sudden appearance or disappearance of a surface. This abrupt (dis)appearance is a catastrophic event, in the sense that the structure of the image is changed qualitatively. In the present corpus, the observer's line of sight is orthogonal to two of the three main axes of the viewing sphere (and therefore parallel with the third axis) in the Front-G, Back-G, Side-G, and Top views. To the extent 
that the surfaces of a particular object are parallel with these axes, the views will be unstable and will tend to be evaluated as the worst views.

In sum, the stable views are generally considered to be better than the unstable views. Note, however, that this preference does not necessarily imply that the brain stores the stable views at the expense of the unstable views. In principle, it is even possible that the unstable views are stored, but subjects express a preference for stable views because these views activate several stored unstable views simultaneously (Perrett, Oram, Hietanen, \& Benson, 1994, pp. 46-47). Moreover, experimental tasks other than goodness-of-view ratings may well reveal other preferences. For instance, Perrett and Harries (1988; also see Harries, Perrett, \& Lavender, 1991, and Perrett, Harries, \& Looker, 1992) recorded the time that subjects spent inspecting different perspective views of novel objects. It was found that the inspection time was longest to the unstable views, from which Perrett and colleagues inferred that the visual system has a preference to store the unstable views. This converges with physiological evidence obtained in singlecell recording studies (Perrett et al., 1991). Future research will have to further unravel the nature of this task dependence and the precise format of the underlying object representations. We hope that the present corpus contributes to this enterprise.

\section{REFERENCES}

AUTODESK, Inc. (1993). Autodesk 3D studio (Release 3). Sausalito, CA: Author.

BARTRAM, D. J. (1976). Levels of coding in picture picture comparison tasks. Memory \& Cognition, 4, 593-602.

Battig, W. F., \& Montague, W. E. (1969). Category norms for verbal items in 56 categories: A replication and extension of the Connecticut category norms. Journal of Experimental Psychology Monographs, 80(3, Pt. 2).

Biederman, I., \& CoOPER, E. E. (1991). Evidence for complete translational and reflectional invariance in visual object priming. Perception, 20, 585-593.

Biederman, I., \& COOPER, E. E. (1992). Size invariance in visual object priming. Journal of Experimental Psychology: Human Perception \& Performance, 18, 121-133.

Biederman, I., \& Gerhardstein, P. C. (1993). Recognizing depthrotated objects: Evidence and conditions for three-dimensional viewpoint invariance. Journal of Experimental Psychology: Human Perception \& Performance, 19, 1162-1182.

Bruce, V., Valentine, T., \& Baddeley, A. (1987). The basis of the $3 / 4$ view advantage in face recognition. Applied Cognitive Psychology, 1 , 109-120.

Bülthoff, H. H., \& Edelman, S. (1992). Psychophysical support for a two-dimensional view interpolation theory of object recognition. Proceedings of the National Academy of Sciences USA, 89, 60-64.

Carlson-Radvansky, L. A., \& Irwin, D. E. (1993). Frames of reference in vision and language: Where is above? Cognition, 46, 223-244.

De Renzi, E., Scotti, G., \& Spinnler, H. (1969). Perceptual and associative disorders of visual recognition: Relationship to the side of the central lesion. Neurology, 19, 634-642.

Dunn-Ranking, P. (1983). Scaling methods. Hillsdale, NJ: Eribaum.

Edelman, S., \& BülthoFf, H. H. (1992). Orientation dependence in the recognition of familiar and novel views of 3D objects. Vision Research, 32, 2385-2400

Ellis, R., \& AllPoRT, D. A. (1986). Multiple levels of representation for visual objects: A behavioural study. In A. G. Cohn \& J. R. Thomas
(Eds.), Artificial intelligence and its applications (pp. 245-257). Chichester: Wiley.

Ellis, R., Allport, D. A., Humphreys, G. W., \& Collis, J. (1989). Varieties of object constancy. Quarterly Journal of Experimental Psychology, 41A, 775-796.

FaRAh, M. J., Rochlin, R., \& Klein, K. L. (1994). Orientation invariance and geometric primitives in shape recognition. Cognitive Science, 18, 325-344.

Garnham, A. (1989). A unified theory of the meaning of some spatial relational terms. Cognition, 31, 45-60.

GuILFORD, J. P. (1954). Psychometric methods (2nd ed.). New York: McGraw-Hill.

Gulliksen, H., \& TuKEy, J. W. (1958). Reliability for the law of comparative judgment. Psychometrika, 23, 95-110.

Harries, M. H., Perrett, D. I., \& Lavender, A. (1991). Preferential inspection of views of 3-D model heads. Perception, 20, 669-680.

Hinton, G. E., \& Parsons, L. M. (1988). Scene-based and viewercentered representations for comparing shapes. Cognition, 30, 1-35.

Howard, I. P. (1982). Human visual orientation. Chichester: Wiley. Humphrey, G. K., \& Jolicoevr, P. (1988). Visual object identification: Some effects of image foreshortening and monocular depth cues. In Z. W. Pylyshyn (Ed.), Computational processes in human vision. An interdisciplinary perspective (pp. 429-442). Norwood. NJ: Ablex

Humphrey, G. K., \& Jolicoevr, P. (1993). An examination of the effects of axis foreshortening, monocular depth cues, and visual field on object identification. Quarterly Journal of Experimental Psychology, 46A, 137-159.

Humphrey, G. K., \& Khan, S. C. (1992). Recognizing novel views of three-dimensional objects. Canadian Journal of Psychology, 46, 170190.

HumPhreys, G. W., \& Quinlan, P. T. (1987). Normal and pathological processes in visual object constancy. In G. W. Humphreys \& M. J. Riddoch (Eds.), Visual object processing: A cognitive neuropsychological approach (pp. 43-105). Hillsdale, NJ: Erlbaum.

Humphreys, G. W., \& RidDoch, M. J. (1984). Routes to object constancy: Implications from neurological impairments of object constancy. Quarterly Journal of Experimental Psychology, 36A, 385-415

HuMPhREYS, G. W., \& RiDDOCH, M. J. (1985). Authors' correction to "Routes to object constancy." Quarterly, Journal of Experimental Psychology, 37A, 493-495.

JolicoEUR, P. (1985). The time to name disoriented natural objects. Memory \& Cognition, 13, 289-303.

Jolicoeur, P. (1988). Mental rotation and the identification of disoriented objects. Canadian Journal of Psychology, 42, 461-478.

Kelter, S., Grötzbach, H., Freiheit, R., Höhle, B., Wutzig, S., \& DiESCH, E. (1984). Object identification: The mental representation of physical and conceptual attributes. Memory \& Cognition, 12, 123133.

Kendall, M. R. (1962). Rank correlation methods (3rd ed.). London: Charles Griffin.

Koenderink, J. J., \& van DoOrn, A. J. (1976). The singularities of the visual mapping. Biological Cybernetics, 24, 51-59.

Koenderink, J. J., \& van Doorn, A. J. (1977). How an ambulant observer can construct a model of the environment from the geometrical structure of the visual inflow. In G. Hauske \& E. Butenandt (Eds.), Kybernetic 1977 (pp. 224-247). Munich: Oldenburg.

Koenderink, J. J., \& VAN DoORN, A. J. (1979). The internal representation of solid shape with respect to vision. Biological Cybernetics, 24, 51-59.

LANGdon, P. M., MaYhew, J. E. W., \& Frisby, J. P. (1991). In search of "characteristic view" 3D object representations in human vision using ratings of perceived differences between views. In J. E. W. Mayhew \& J. P. Frisby (Eds.), $3 D$ model recognition from stereoscopic views (pp. 244-248). Cambridge, MA: MIT Press.

LAyman, S., \& GreEn, E. (1988). The effect of stroke on object recognition. Brain \& Cognition, 7, 87-114.

LEVELt, W. J. M. (1984). Some perceptual limitations on talking about space. In A. J. van Doorn, W. A. van de Grind, \& J. J. Koenderink (Eds.), Limits in perception (pp. 323-358). Utrecht, The Netherlands: VNU Science Press. 
MARR, D., \& NishiHARA, H. K. (1978). Representation and recognition of the spatial organization of three-dimensional shapes. Proceedings of the Royal Society of London, B200, 269-294.

MarShall, E., \& WALKER, P. (1987). Visual memory for pictorial stimuli in a serial choice reaction-time task. British Journal of $P$ sychology, 78, 213-231.

Metzler, J., \& Shepard, R. N. (1974). Transformational studies of the internal representation of three-dimensional objects. In R. Solso (Ed.) Theories in cognitive psychology: The Loyola symposium (pp. 147201). Potomac, MD: Erlbaum.

Milligan, G. W., \& CoOper, M. C. (1985). An examination of procedures for determining the number of clusters in a data set. Psychometrika, 50, 159-179.

MOSTELLER, F. (1951). Remarks on the method of paired comparisons: III A test of significance for paired comparisons when equal standard deviates and equal correlations are assumed. Psychometrika, 16, 207- 218

Palmer, S., Rosch, E., \& Chase, P. (1981). Canonical perspective and the perception of objects. In J. Long \& A. Baddeley (Eds.), Attention and performance $I X(\mathrm{pp} .135-151)$. Hillsdale, $\mathrm{NJ}$ : Erlbaum.

PARSONS, L. M. (1987a). Imagined spatial transformations of one's body. Joumal of Experimental Psychology: General, 116. 172-191.

PARSONs, L. M. (1987b). Imagined spatial transformations of one's hands and feet. Cognitive Psychology, 19, 178-241.

PerretT, D. I., \& Harries, M. H. (1988). Characteristic views and the visual inspection of simple faceted and smooth objects: "Tetrahedra and potatoes." Perception, 17, 703-720.

Perrett, D. I., Harries, M. H., \& LoOker, S. (1992). Use of preferential inspection to define the viewing sphere and characteristic views of an arbitrary machined tool part. Perception, 21, 497-515.

Perretr, D. I., Oram, M. W., Harries, M. H., Bevan, R., Hietanen, J. K., Benson, P. J., \& Thomas, S. (1991). Viewer-centered and objectcentered coding of heads in the macaque temporal cortex. Experimental Brain Research, 86, 159-173.

Perrett, D. I., Oram, M. W., Hietanen, J. K., \& Benson, P. J. (I994) Issues of representation in object vision. In M. J. Farah \& G. Ratcliff (Eds.), The newropsychology of high-level vision: Collected tutorial essays (pp. 33-61). Hillsdale, NJ: Erlbaum

ROBERTS, T., \& BkUCE, V. (1989). Repetition priming of face recognition in a serial choice reaction-time task. British Joumal of Pswcholog! , 80, 201-211.

Rock, L., \& DiVITA, J. (1987). A case of viewer-centered object perception. Cognitive Psichology, 19, 280-293.

RoCK, L., WHEELER, D., \& TUDOR, L. (1989). Can we imagine how objects look from other viewpoints? Cognitive Psychology, 21, 185-210

SAS InstITUTE, INC. (1990). SAS/STAT user's guide (Version 6). Cary, NC: Author.

Shepard, R. N., \& Metzl.er, J. (1971). Mental rotation of threedimensional objects. Science, 171, 701-703.

SNODGRaSS, J. G., \& VANDERWART, M. (1980). A standardized set of 260 pictures: Norms for name agreement, image agreement, familiarity, and visual complexity. Journal of Experimental Psychology: Human Learning \& Memory, 6, 174-215.

SRINIVAS, K. (1993). Perceptual specificity in nonverbal priming. Journal of Experimental Psychology: Leaming, Memors: \& Cognifion, 19, 582-602.

Thurstone, L. L. (1927). A law of comparative judgment. Psichological Review, 34, 273-286.

TORGERSon, W. S. (1958). Theomy and methods of scaling. New York: Wiley.

Verfallt.te, K. (1992). Variant points of view on viewpoint invariance. Canadian Journal of Psychologv, 46, 21 5-235.

VerfallLIe, K. (1993). Orientation-dependent priming effects in the perception of biological motion. Journal of Experimental Psychology: Human Perception \& Performance, 19, 992-1013.

Verfaill.ie, K., De Troy, A., \& Van Rensbergen, J. (1994). Transsaccadic integration of biological motion. Joumal of Experimental Psychology: Learning. Memony, \& Cognition, 20, 649-670.

WARRINGION, E. K., \& JAMES, M. (1986). Visual object recognition in patients with right-hemisphere lesions: Axes or features? Perception $15,355-366$.

Warrington, E. K., \& TAYlor, A. M. (1973). The contribution of the right parietal lobe to object recognition. Cortex, 9, 152-164.

\section{NOTES}

1. There are exceptions. For instance, Hinton and Parsons (1988) used 3-D wooden replicas of the famous objects that Shepard and Metzler (1971) introduced in their seminal work on mental rotation. Other authors also made use of novel objects. Humphrey and Kahn (1992, Experiment 3) constructed novel clay objects, Rock and DiVita (1987) and Rock, Wheeler, and Tudor (1989) presented wire-frame objects (also see Farah, Rochlin, \& Klein, 1994), and Perrett et al. (1992) used an arbitrary machined tool part. Sorne studies used familiar instead of novel objects (e.g., Palmer et al., 1981). Warrington and James (1986) presented subjects with 3-D shadow images of scale models of 10 common objects.

2. The main advantage of this procedure is that the subjects' task is much easier than in a procedure in which subjects directly rate the goodness of view (as in the study of Palmer et al., 1981). In our experiment, the subjects' task implies only an ordinal judgment. Yet, on the basis of scaling, we can derive an interval scale. Asking subjects a direct rating requires a judgment on an interval scale, which is a much less straightforward task psychologically.

3. Seven objects have 9 different views. For five of these objects (boiler, comb, suitcase, table, and toaster), the back is identical to the front (Front-G and Front-E are identical to Back-G and Back-E, respectively), because the objects are reflectionally symmetrical across the midfrontal plane. There are two objects (funnel and pencil) for which the Side-G, Side- $E$, and Top views are identical. The funnel is rotationally symmetrical around the z-axis, and the pencil has eight planes of symmetry that include the z-axis. One object (rolling pin) combines the characteristics of these two classes of objects, so that there are only 7 different views: Front $-\mathrm{G}$ and Front- $\mathrm{E}$ are identical to Back- $\mathrm{G}$ and Back-E, respectively, because the object's front is identical to its back, and Side-G, Side-E, and top are identical because the object is rotationally symmetrical around the $z$-axis. Because the ashtray has multiple planes of symmetry, the original 11 views reduce to 5 : The $3 \mathrm{G}$ views in which the observer's line of sight coincides with the $x$-axis or $z$-axis (Front-G, Back-G, and Side-G) are indistinguishable, as are the corresponding $3 \mathrm{E}$ views (Front-E, Back-E, and Side-E). The $23 / 4 \mathrm{G}$ views ( $3 / 4$ Front $-\mathrm{G}$ and $3 / 4$ Back-G) are also identical, as are the $23 / 4 \mathrm{E}$ views (3/4 Front-E and $3 / 4$ Back-E). Finally, there are four mono-oriented objects with only 3 views (bottle. glass, saucer, and vase). Because the objects are rotationally symmetrical around the $v$-axis, neither the $5 \mathrm{G}$ views (Front-G, 3/4 Front$G$, Side-G, $3 / 4$ Back-G, and Back-G) nor the $5 \mathrm{E}$ views (Front-E, 3/4 Front-E, Side-E, $3 / 4$ Back-E, and Back-E) can be distinguished.

4. Some views of five of the 9 -view objects (boiler, comb, suitcase, table, and toaster) are mirror reflections of each other. For instance, the $3 / 4$ Front-E view is the mirror image of the $3 / 4$ Back-E view. Nevertheless, we included these views in the study, because they allowed us to explore whether there would be a systematic preference for one lateral orientation over another. A similar test with the 11-view objects would have inflated the total number of views drastically (and therefore the number of pairs of views to be rated in the paired-comparison experiment). We observed no preference for views in which the object's axis of elongation is oriented toward the right over views in which the axis is orjented toward the left. Therefore, we will not come back to this aspect of the experiment.

5. As already mentioned in the Method section, there is no straightforward way to place poly-oriented objects in the viewing sphere, by the very definition of "poly-orientedness" itself. Consequently, as far as the poly-oriented objects are concerned, the cluster analysis is arbitrary to some degree, because the labeling of the views (and therefore the way these objects are entered in the cluster analysis) is determined by the objects' orientation in the viewing sphere. For instance, the poly-oriented objects of the 5th cluster (the axe, the saw, the knife, and the biro) could have been located in the viewing sphere in such a way that they are flat in the midtransverse plane. In this case, they would have been very similar to the objects that are now included in the 4 th cluster. On the other hand even for poly-oriented objects. the location in the viewing sphere was not completely arbitrary (see Method section). The procedure we adopted maximizes symmetry across the midsagittal plane. Indeed, if the poly-oriented objects of the 5th cluster are located in the viewing sphere in such a way that they are flat in the midtransverse plane, they are less symmetrical across the midsagittal plane than are the objects in the 4 th cluster 


\section{APPENDIX A}

Black-and-White, Low-Resolution Renderings of the Different Perspective Views and Their Location on the Goodness-of-View Scale for the 70 Objects

The objects are ordered by their number of views, starting with the 11-view objects and ending with the 3-view objects. The order of presentation of the 11 -view objects is determined by cluster membership, starting with Cluster 1 and ending with Cluster 8 . Within each cluster, the objects are ordered as a function of the degree of cluster membership.

\section{1-VIEW OBJECTS}

\section{Cluster 1}
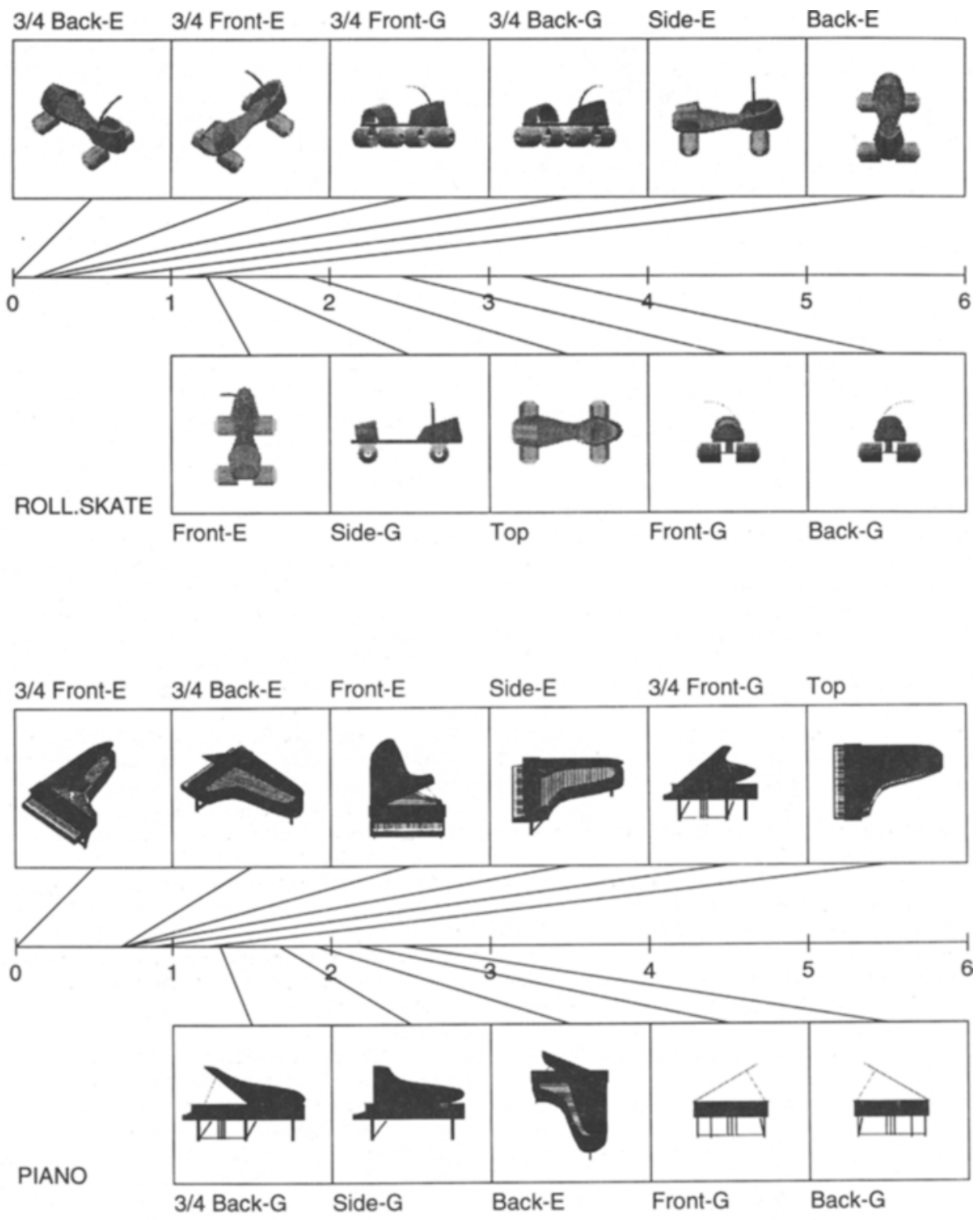
APPENDIX A (Continued)
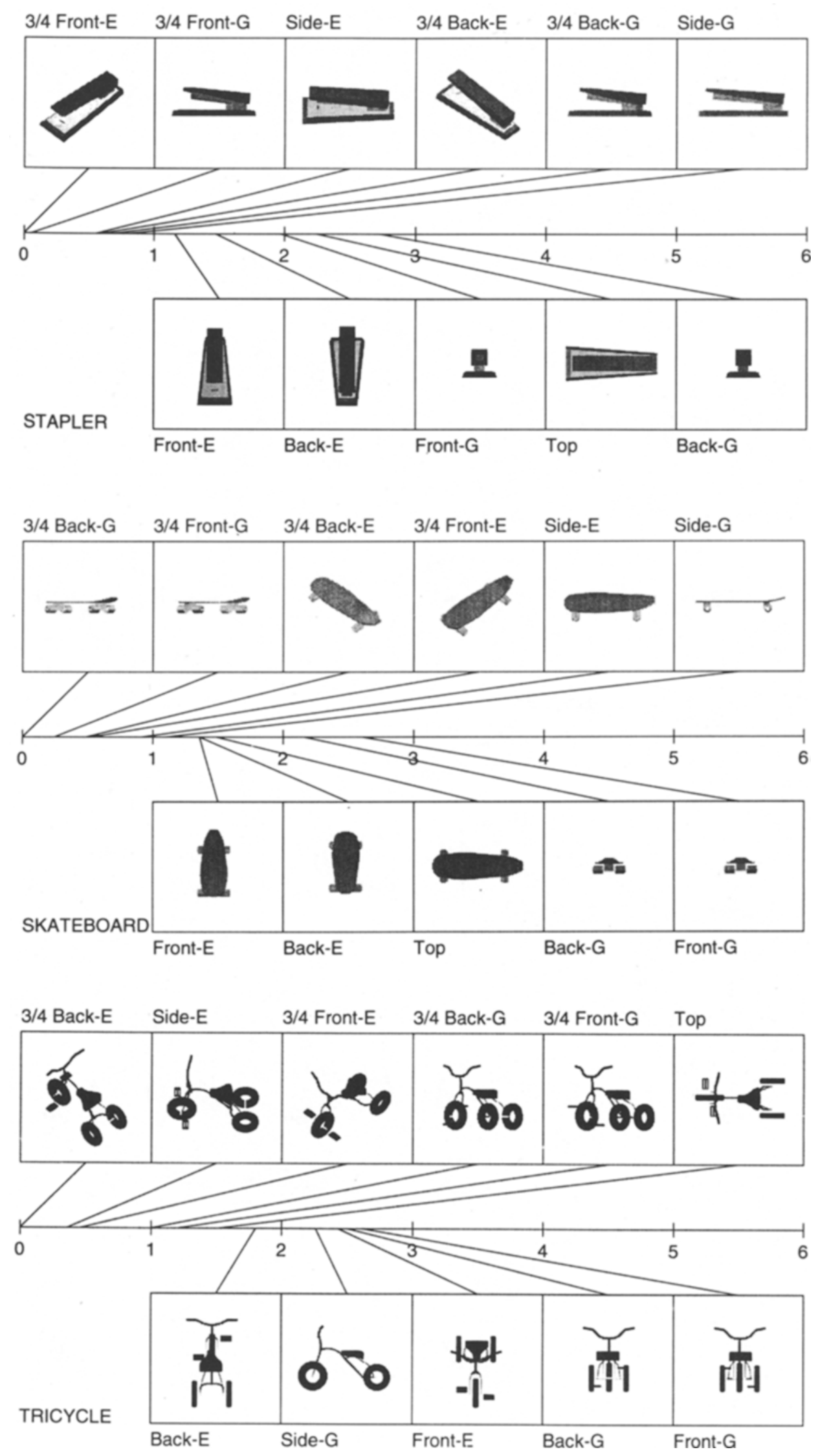
APPENDIX A (Continued)
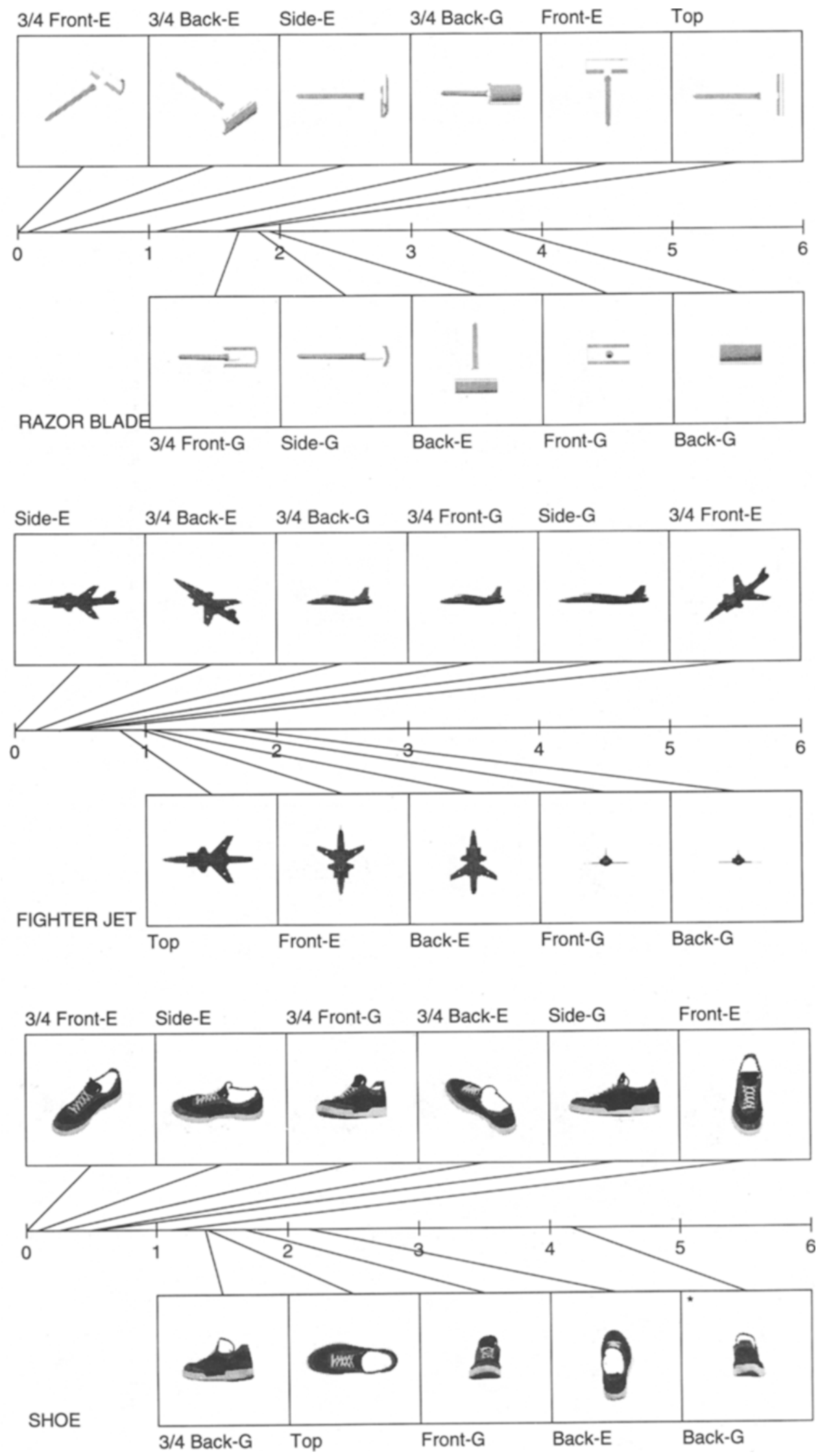
APPENDIX A (Continued)
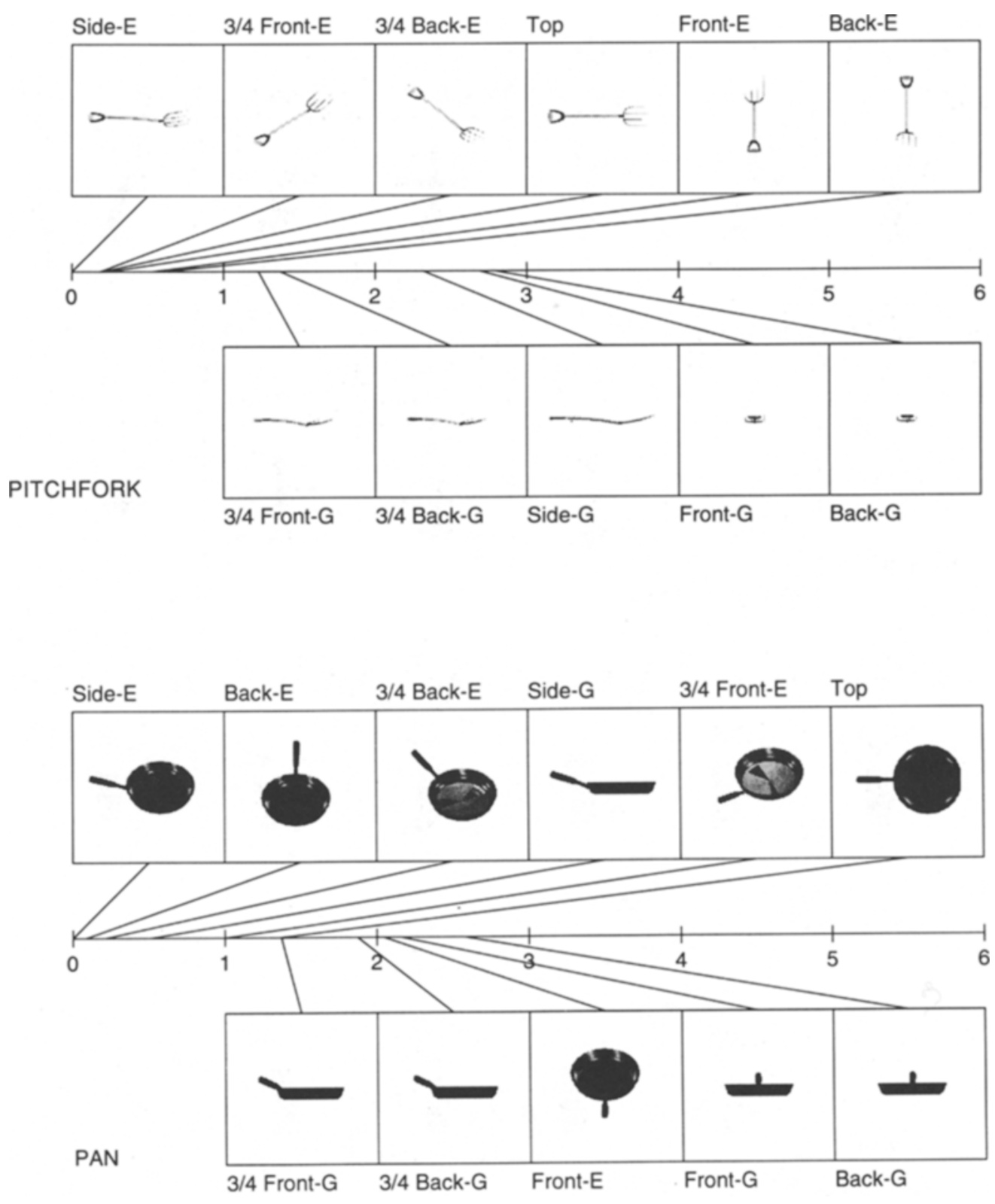


\section{Cluster 2}
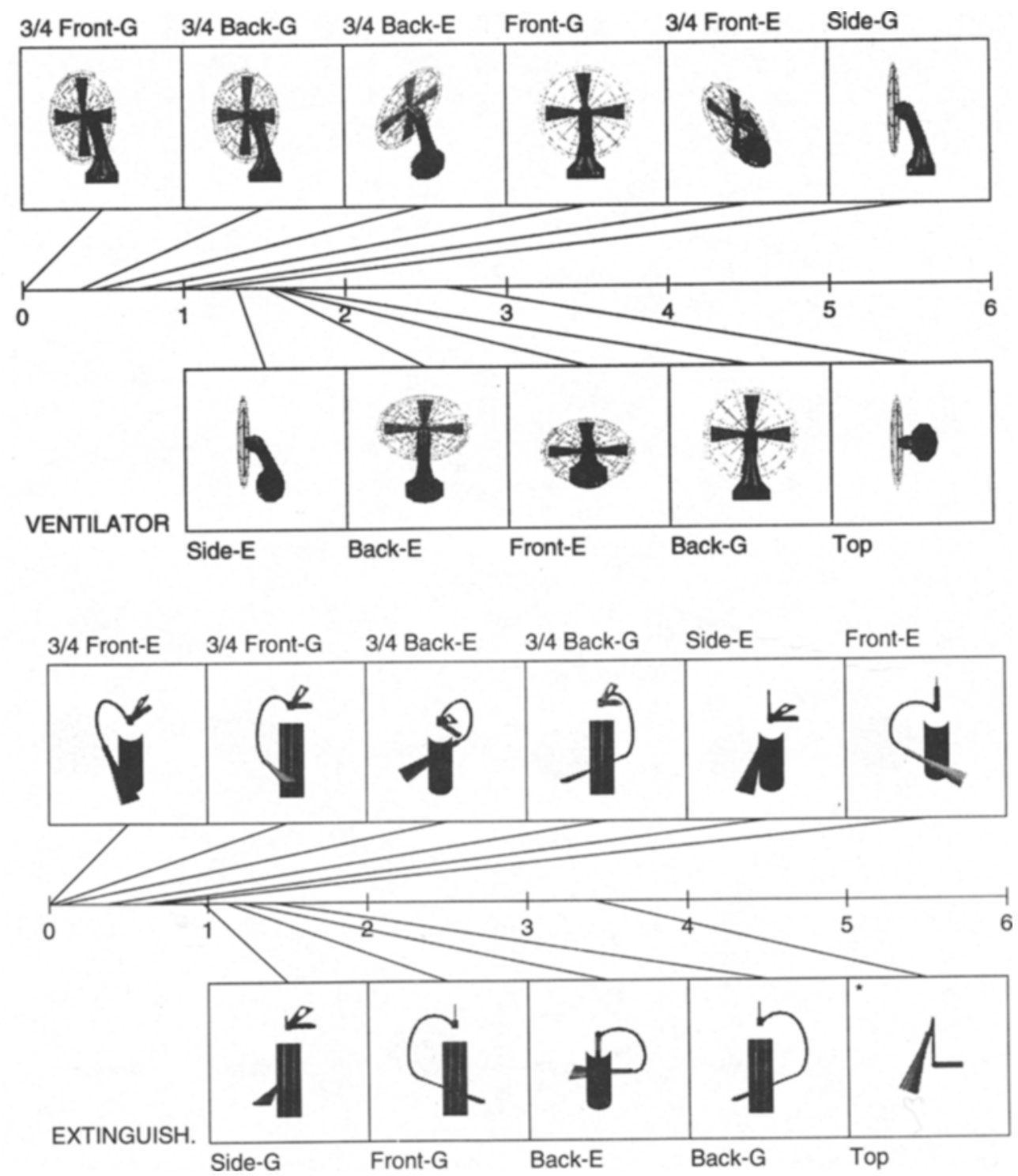
APPENDIX A (Continued)
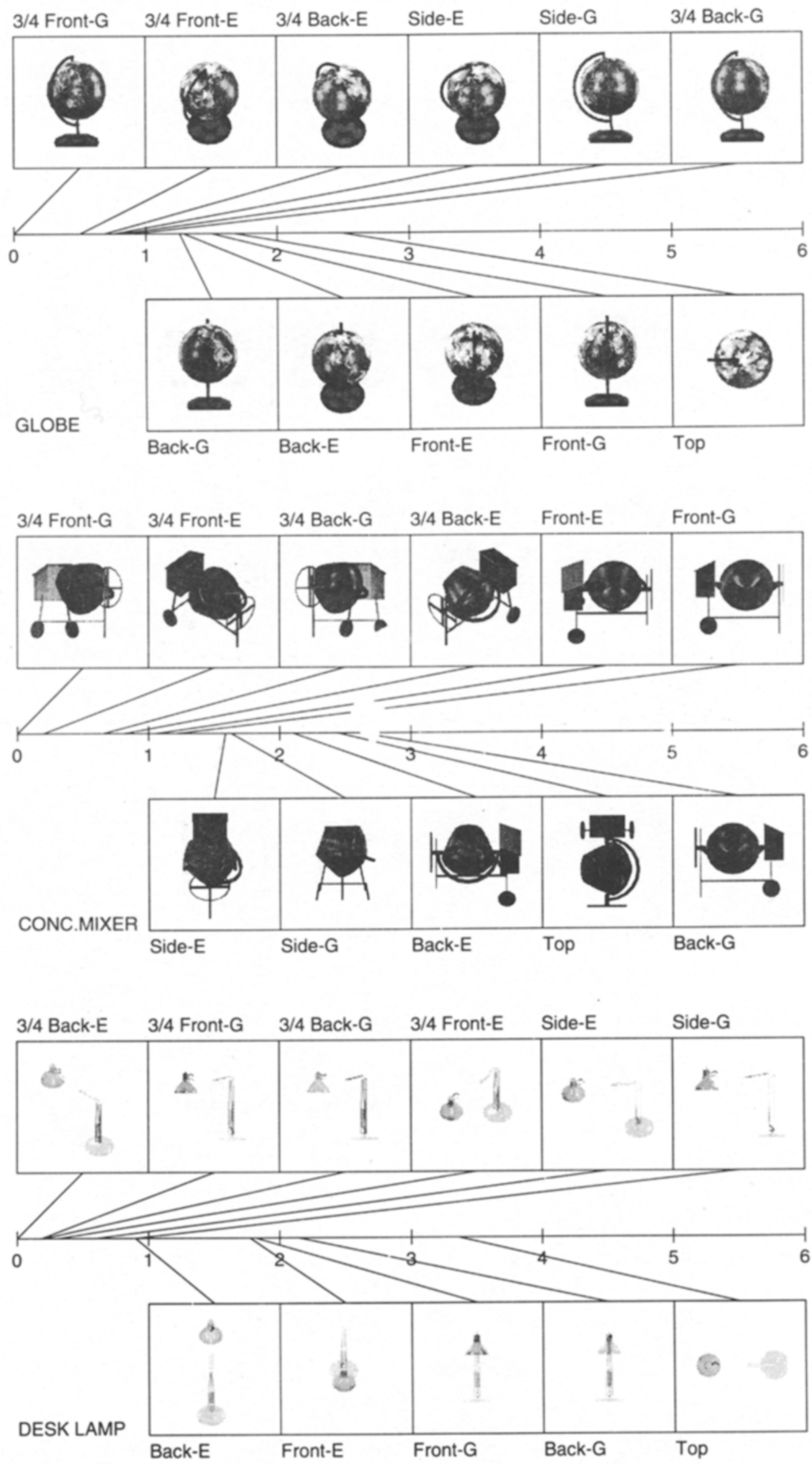
APPENDIX A (Continued)
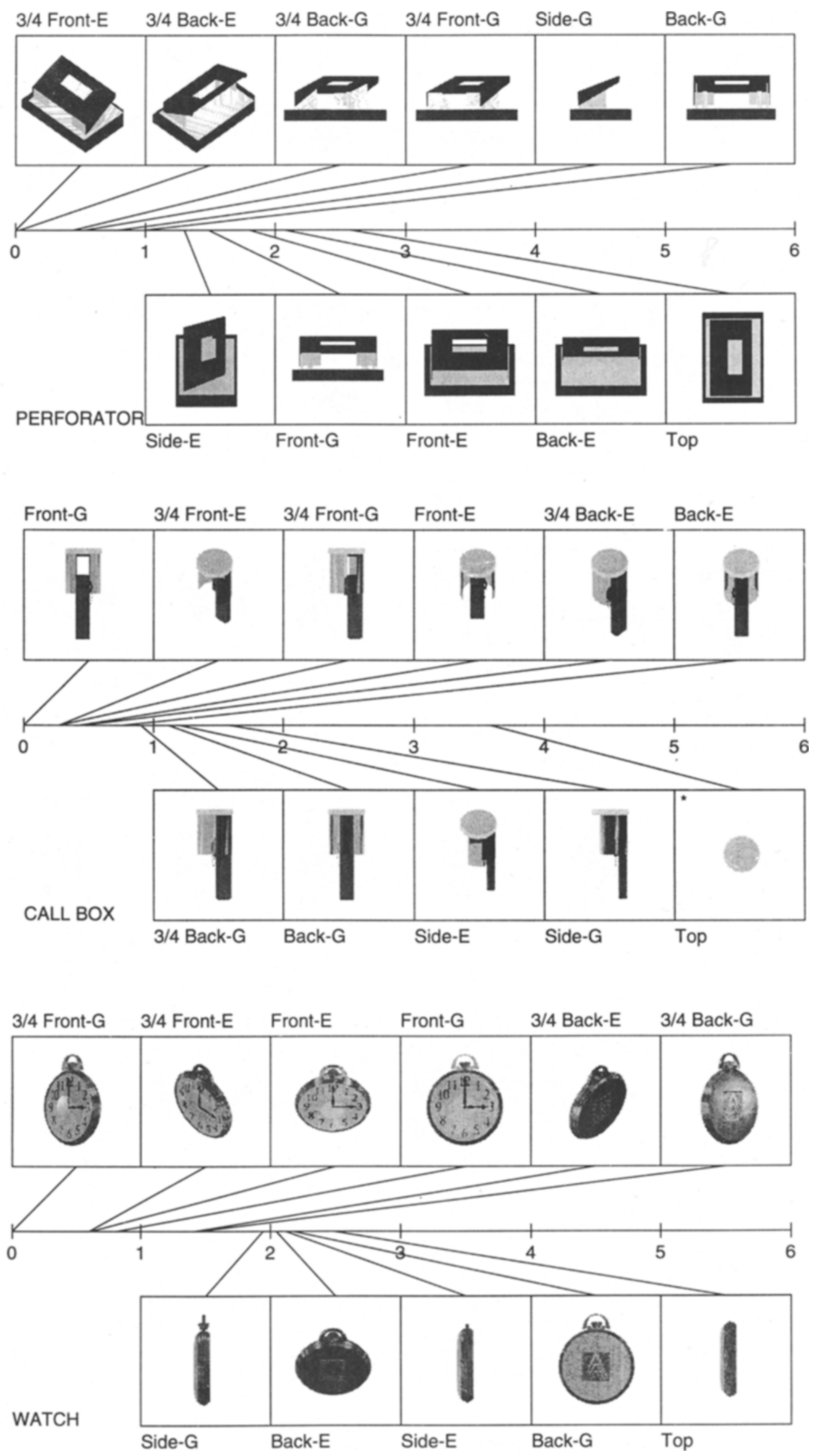


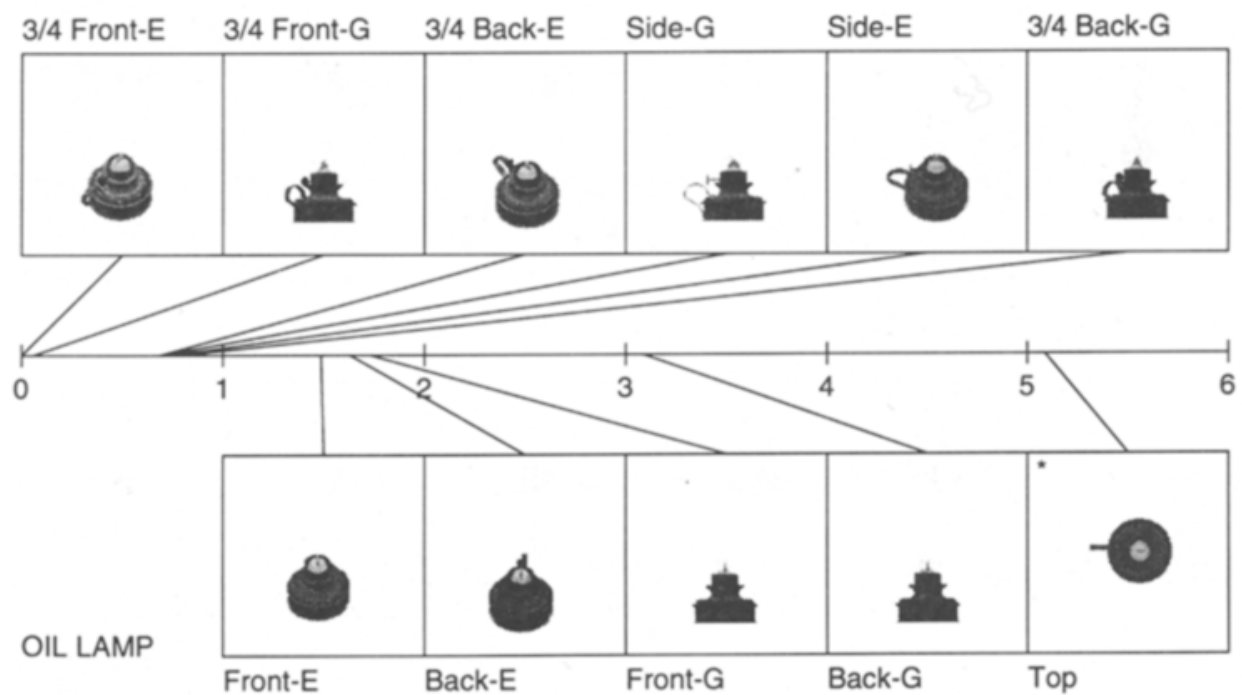

\section{Cluster 3}

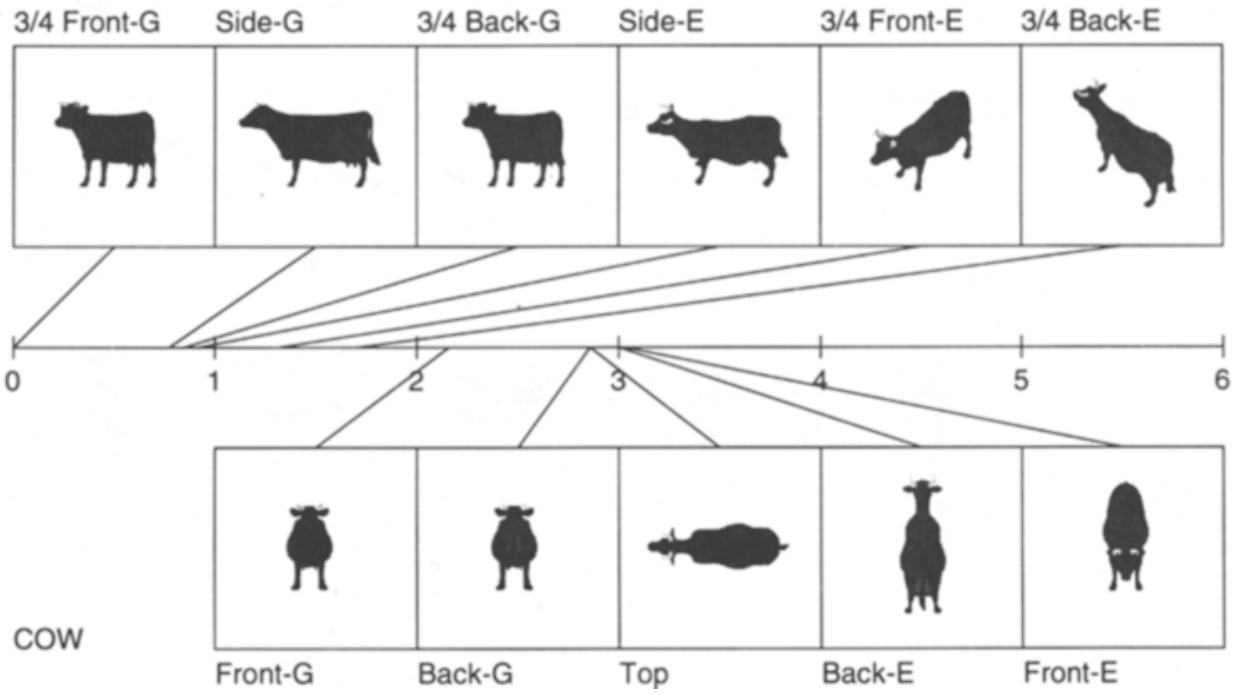



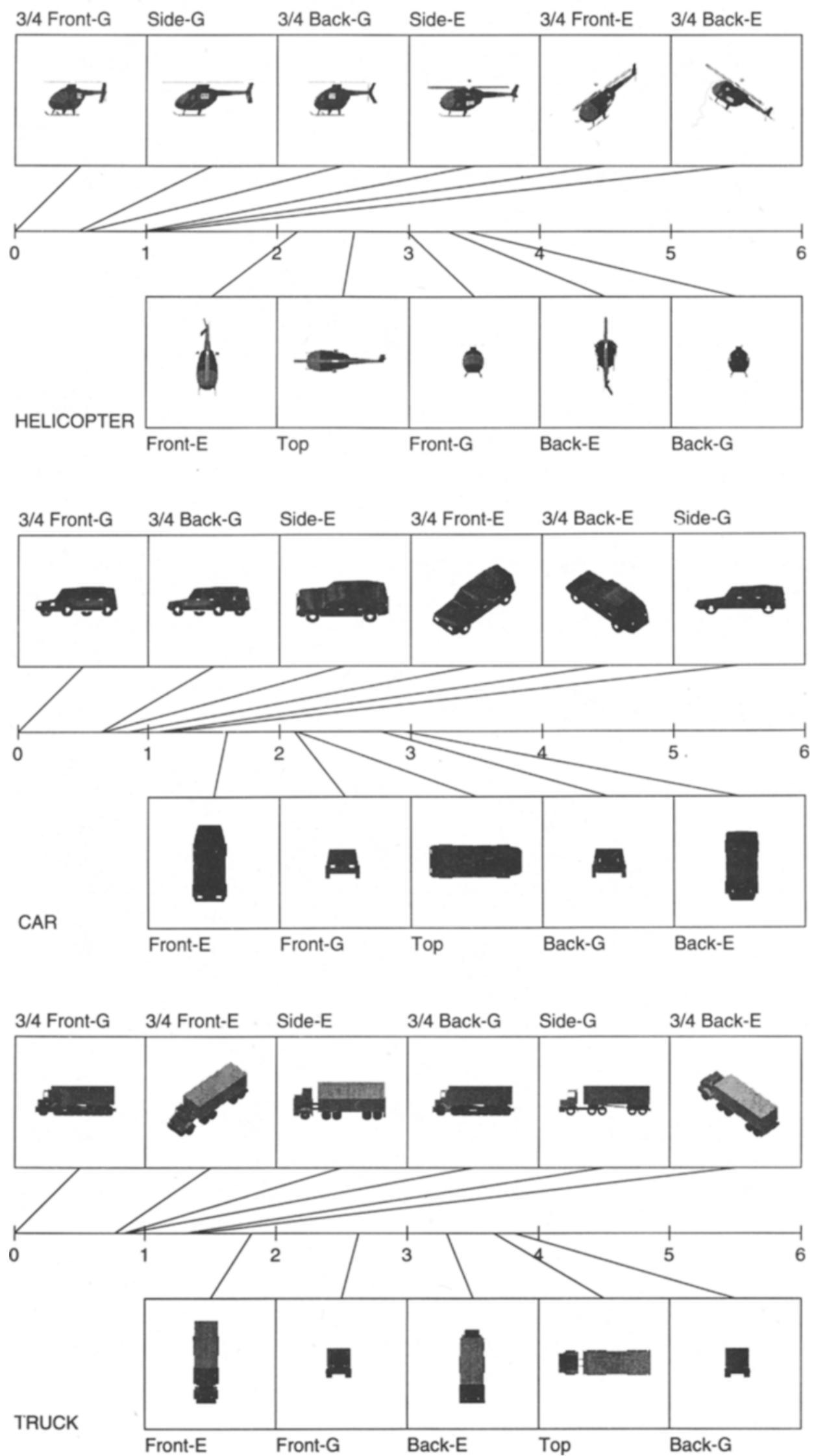
APPENDIX A (Continued)
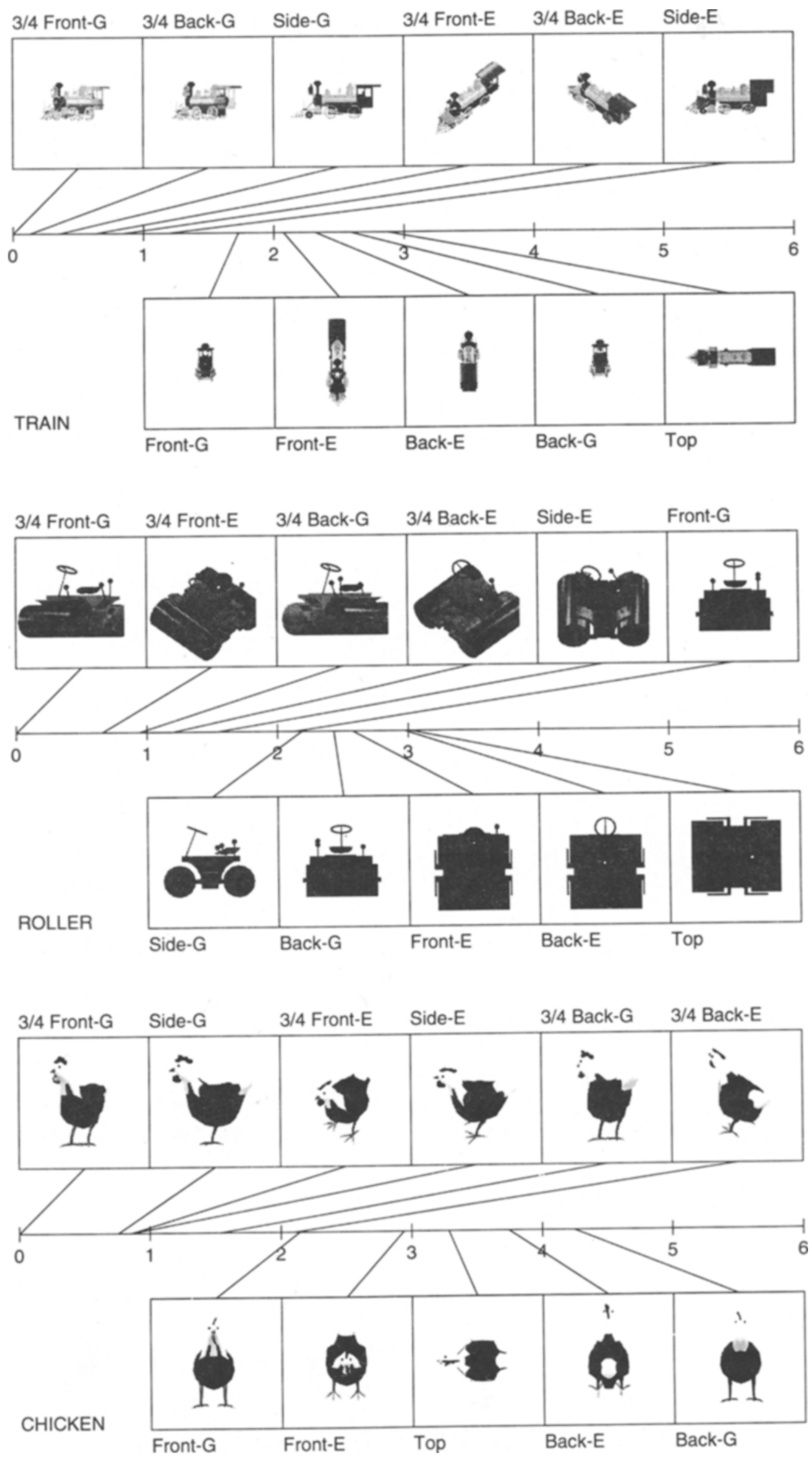
APPENDIX A (Continued)
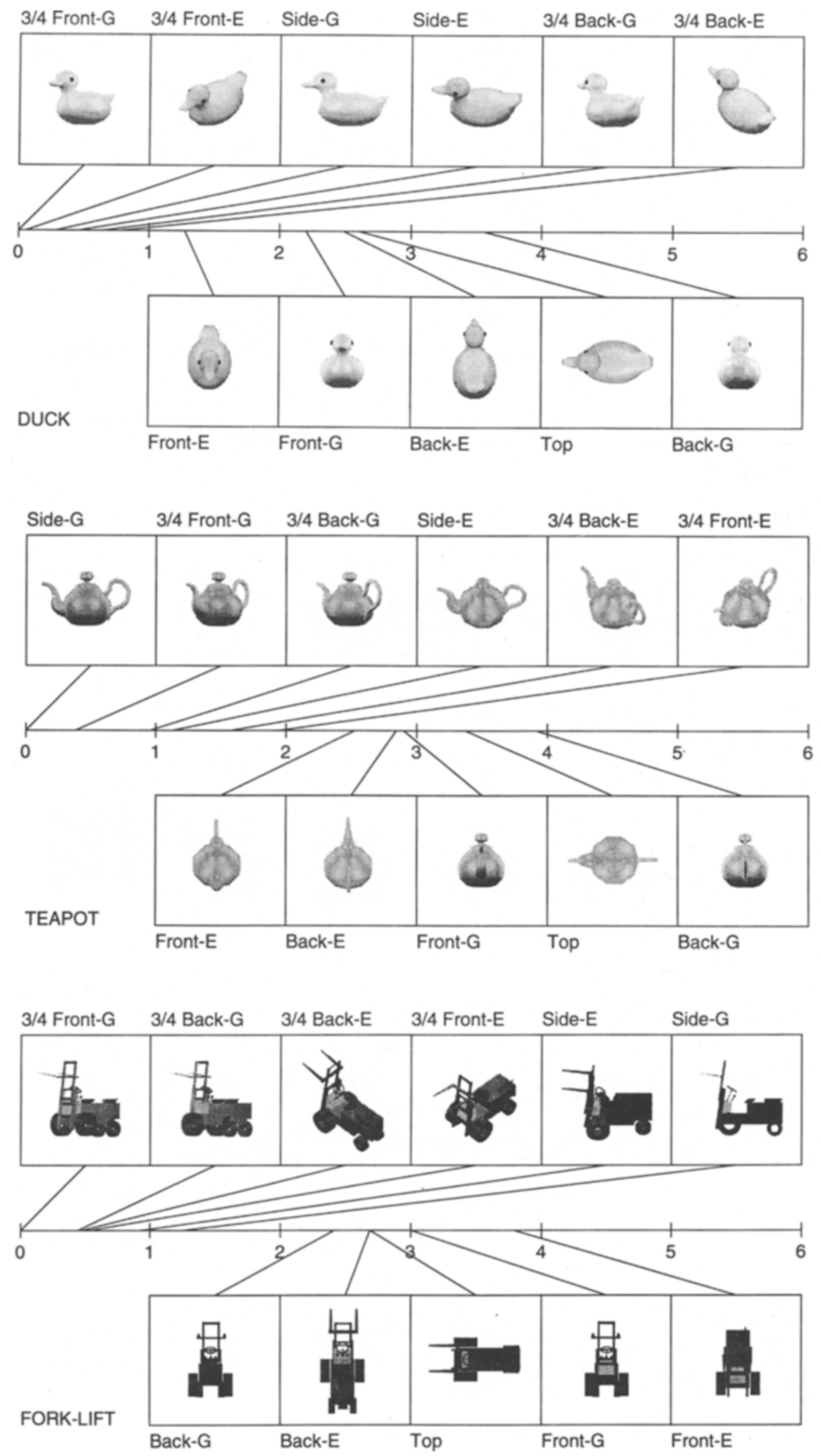


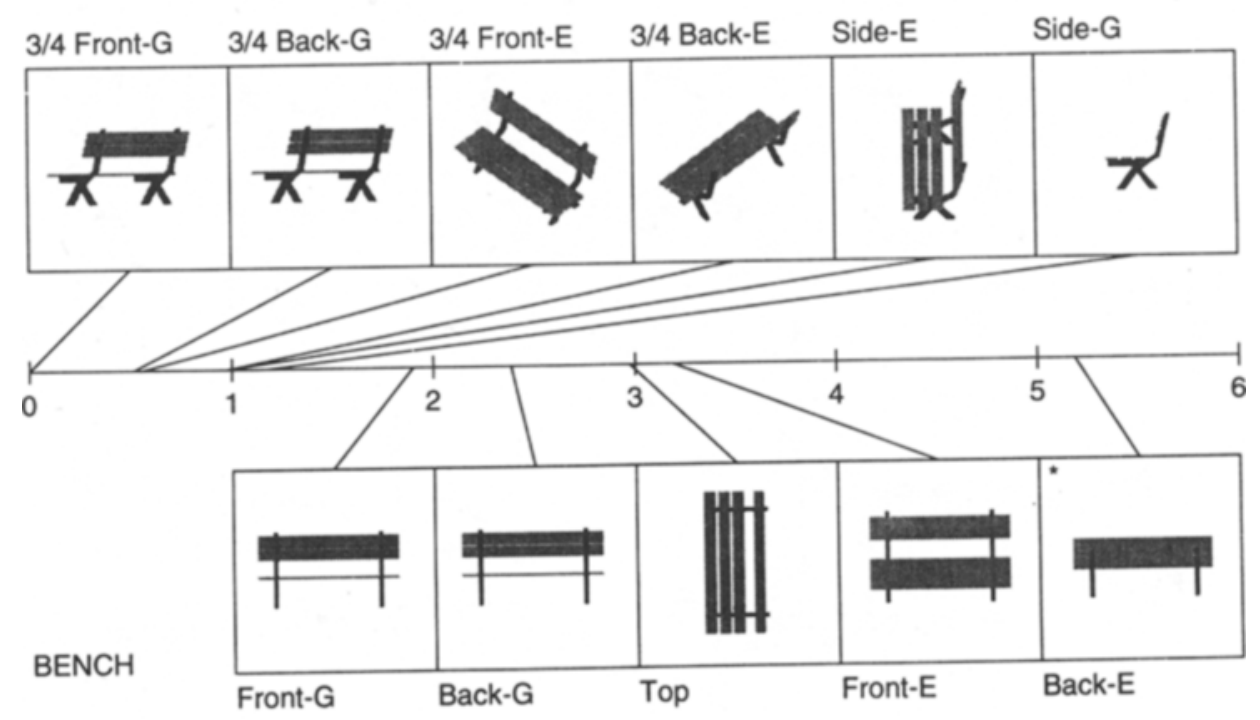

\section{Cluster 4}

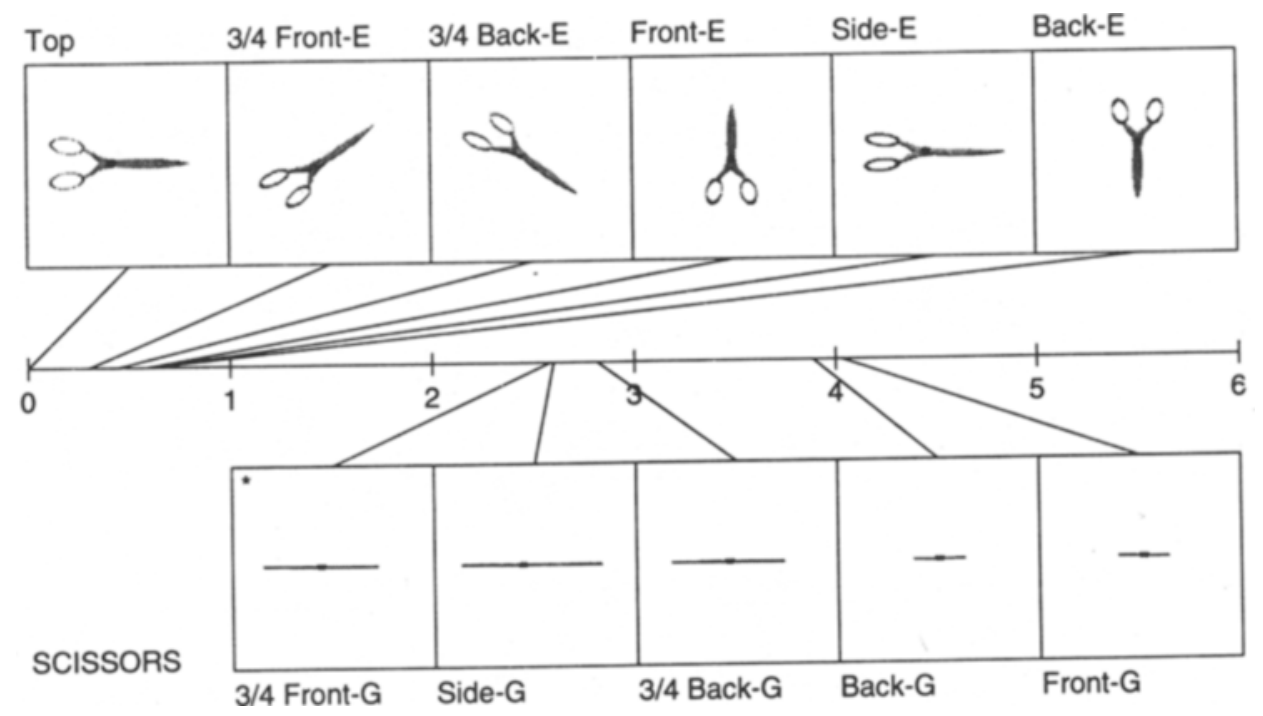



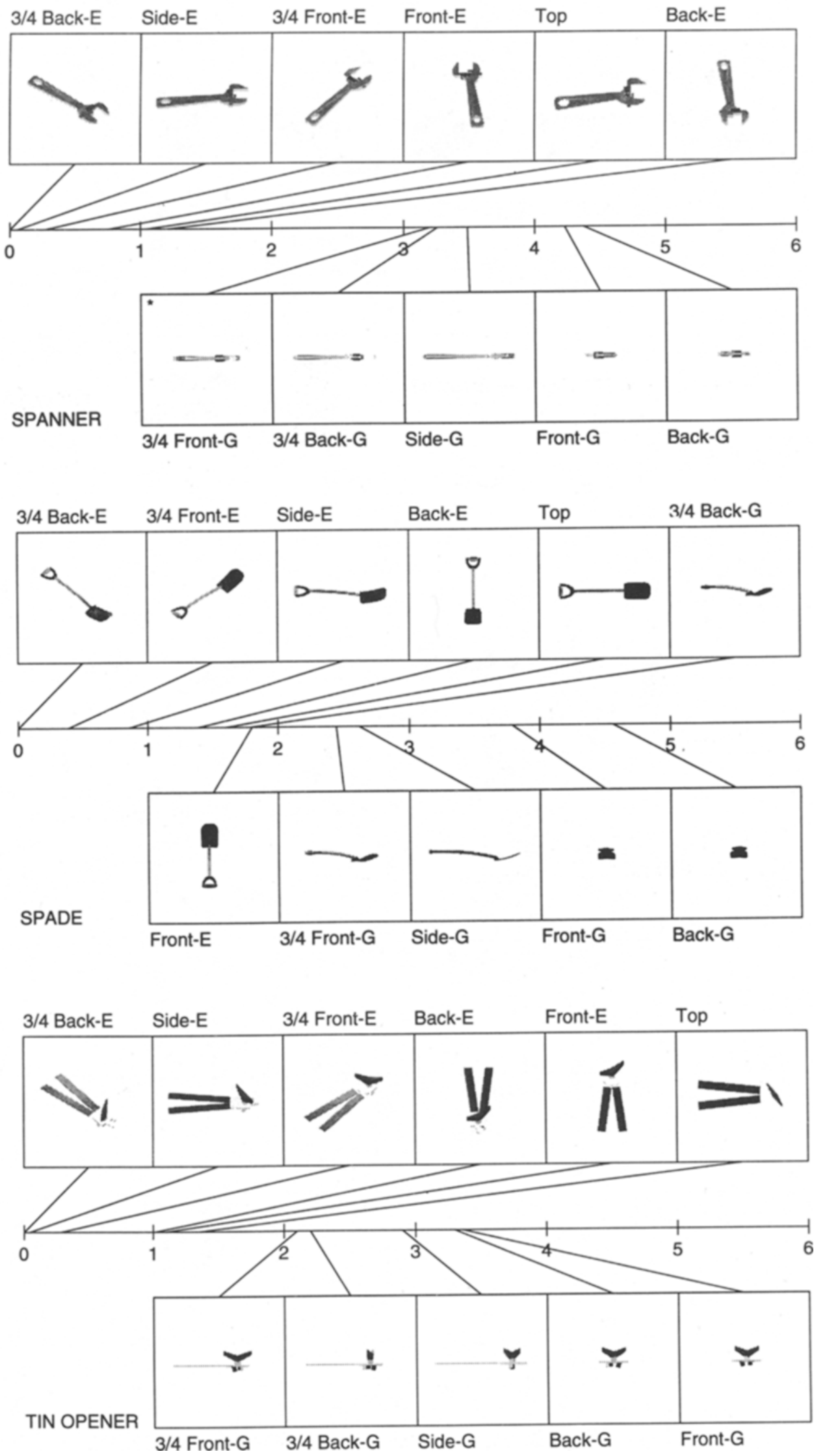
APPENDIX A (Continued)
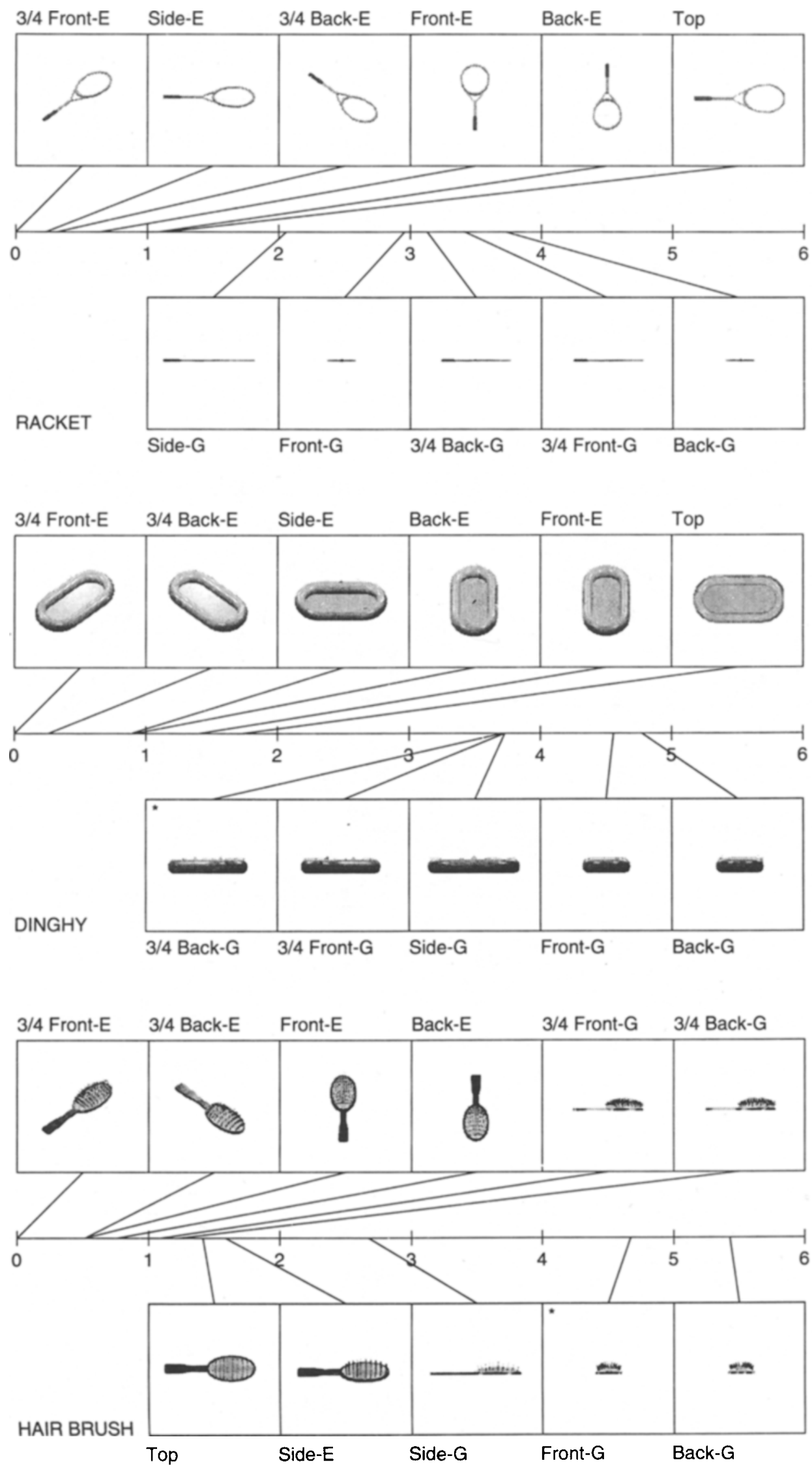

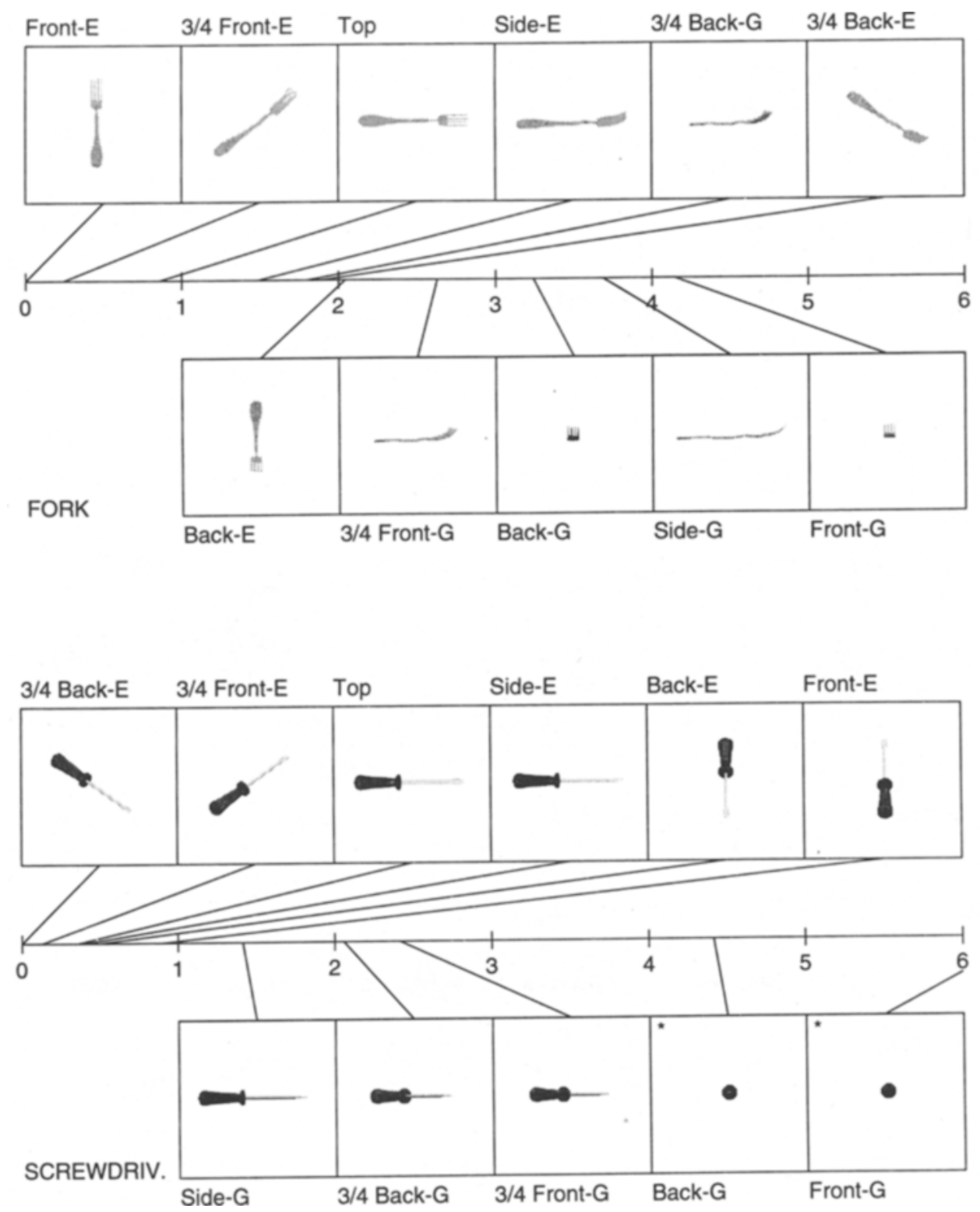


\section{Cluster 5}
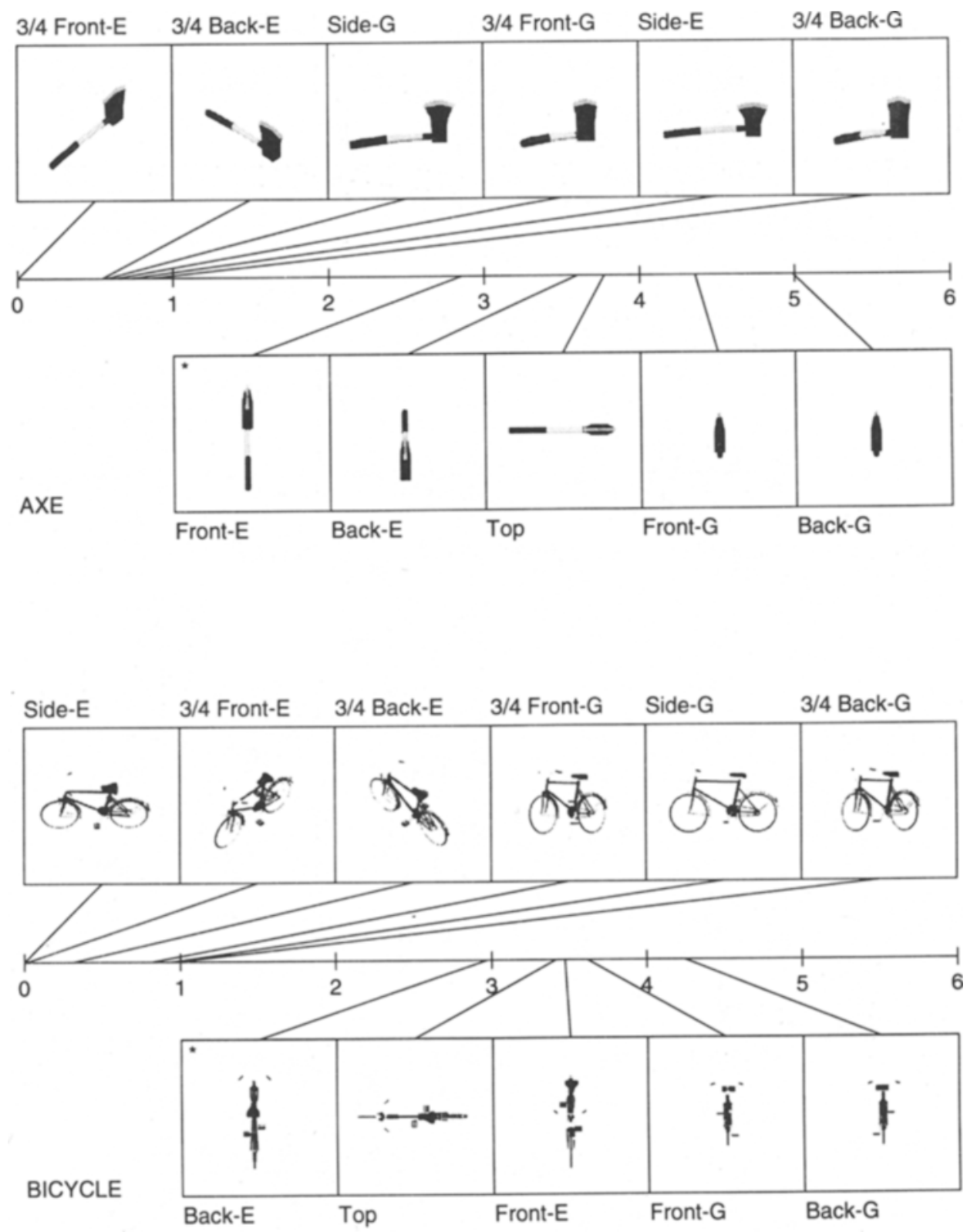
APPENDIX A (Continued)
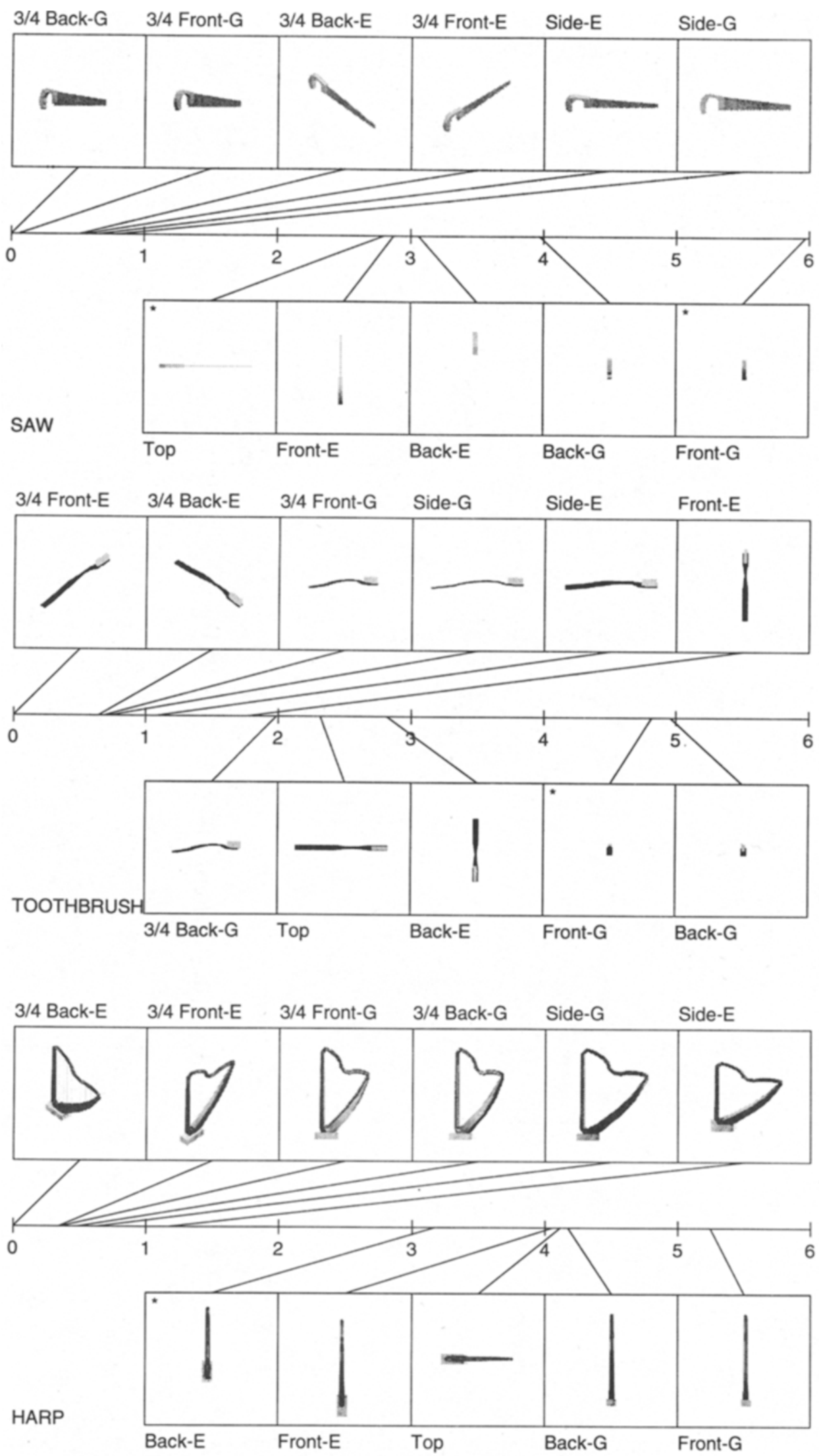
APPENDIX A (Continued)
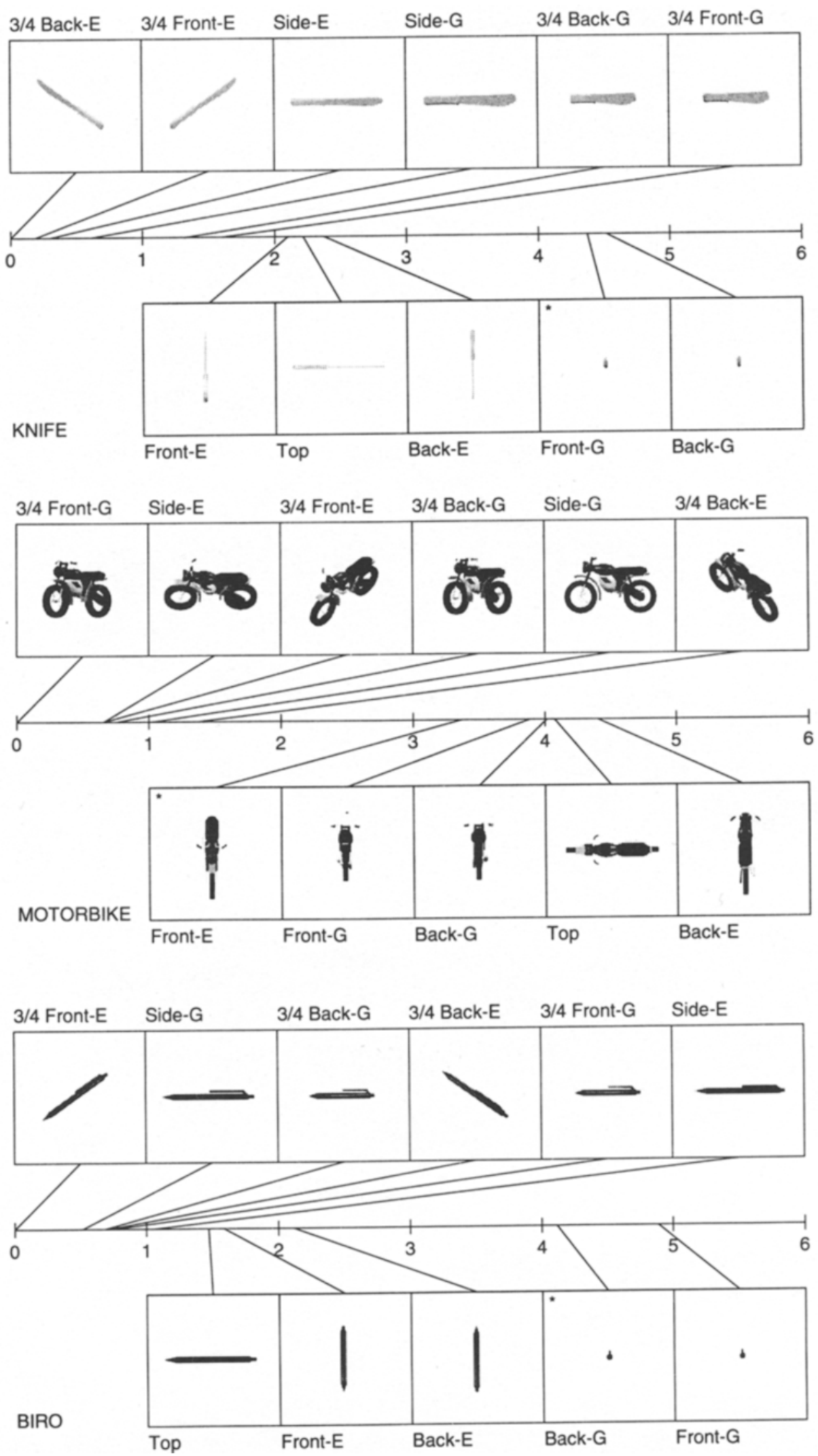
APPENDIX A (Continued)

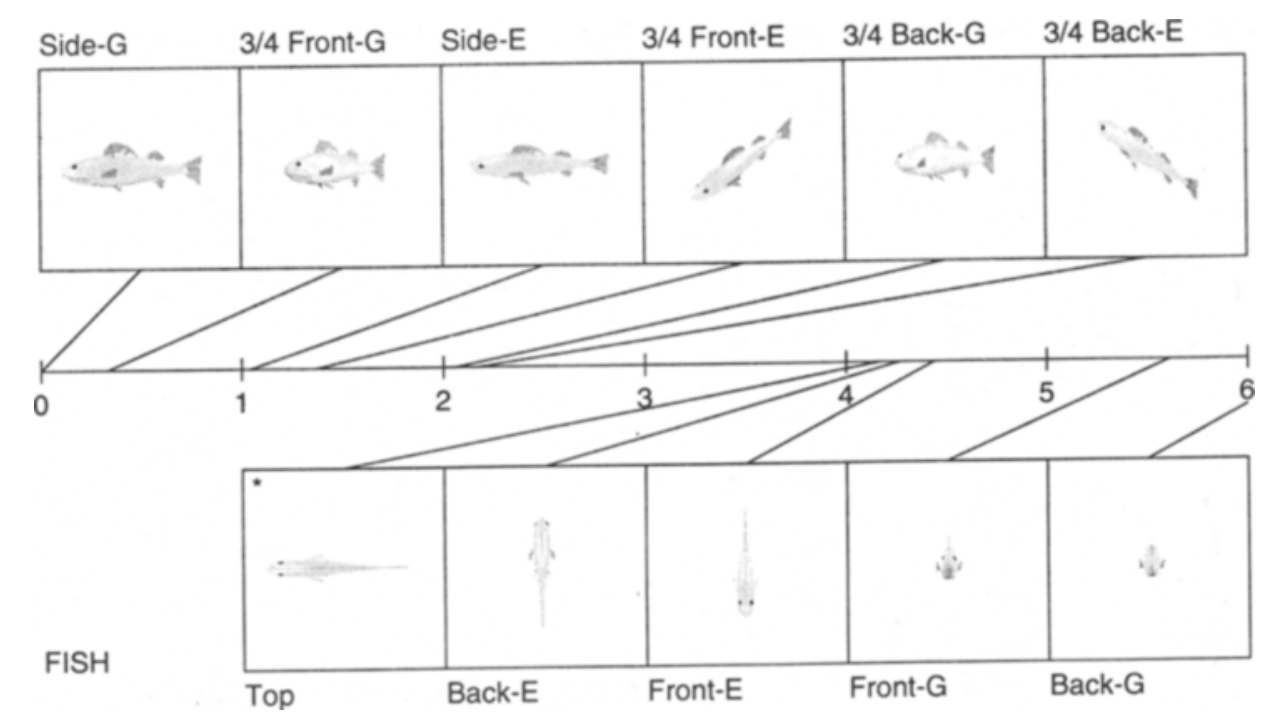

\section{Cluster 6}

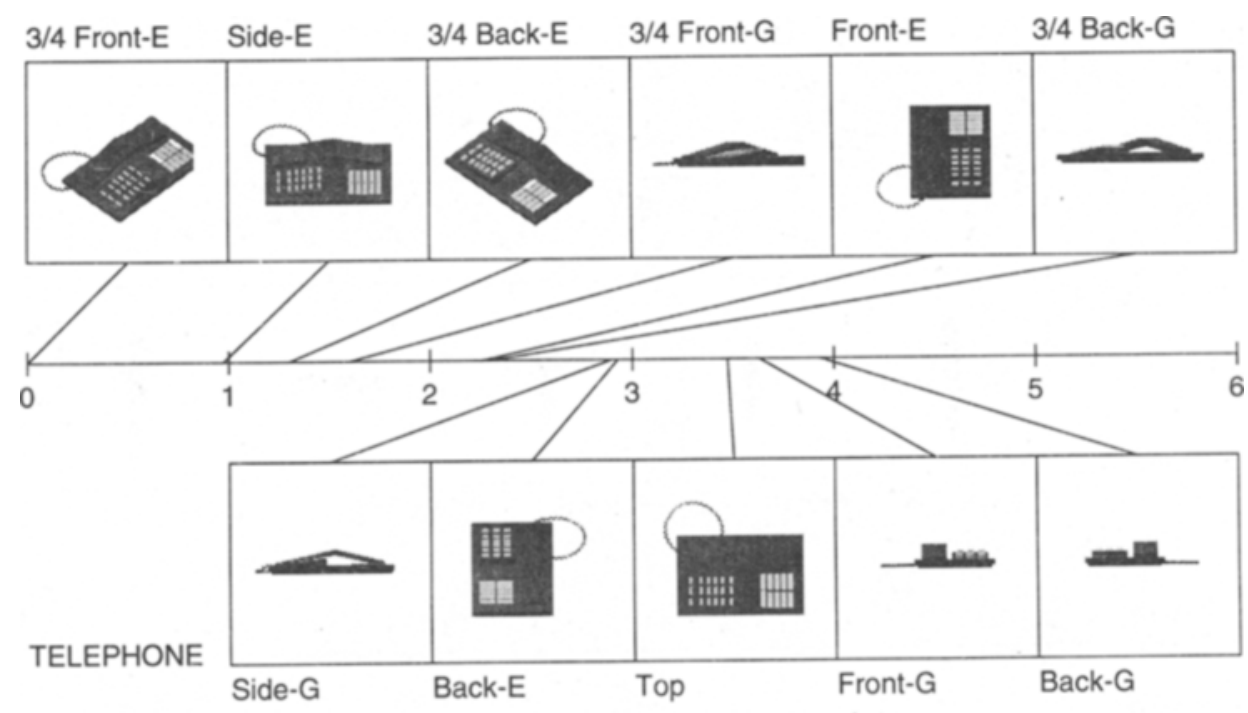



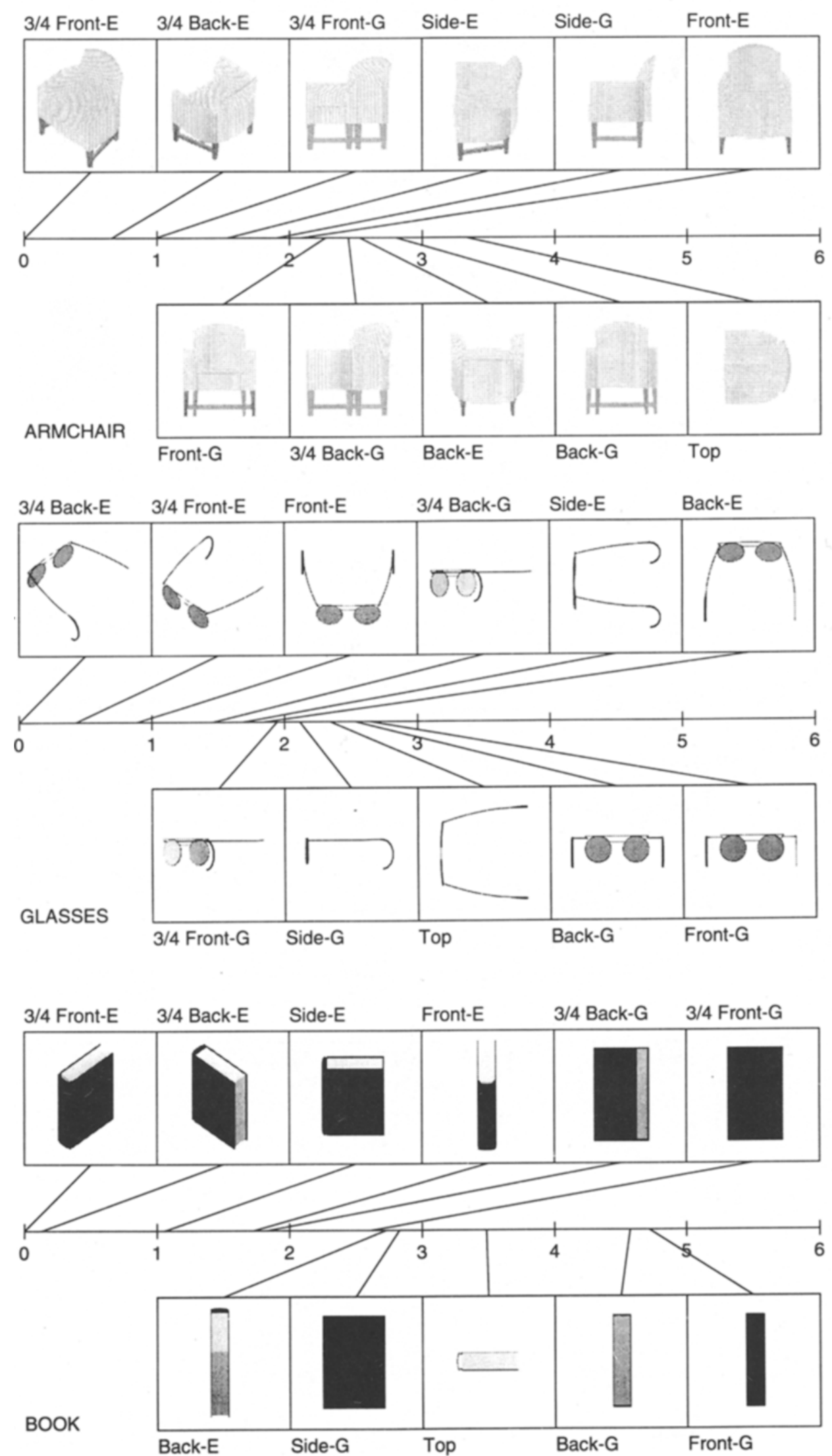


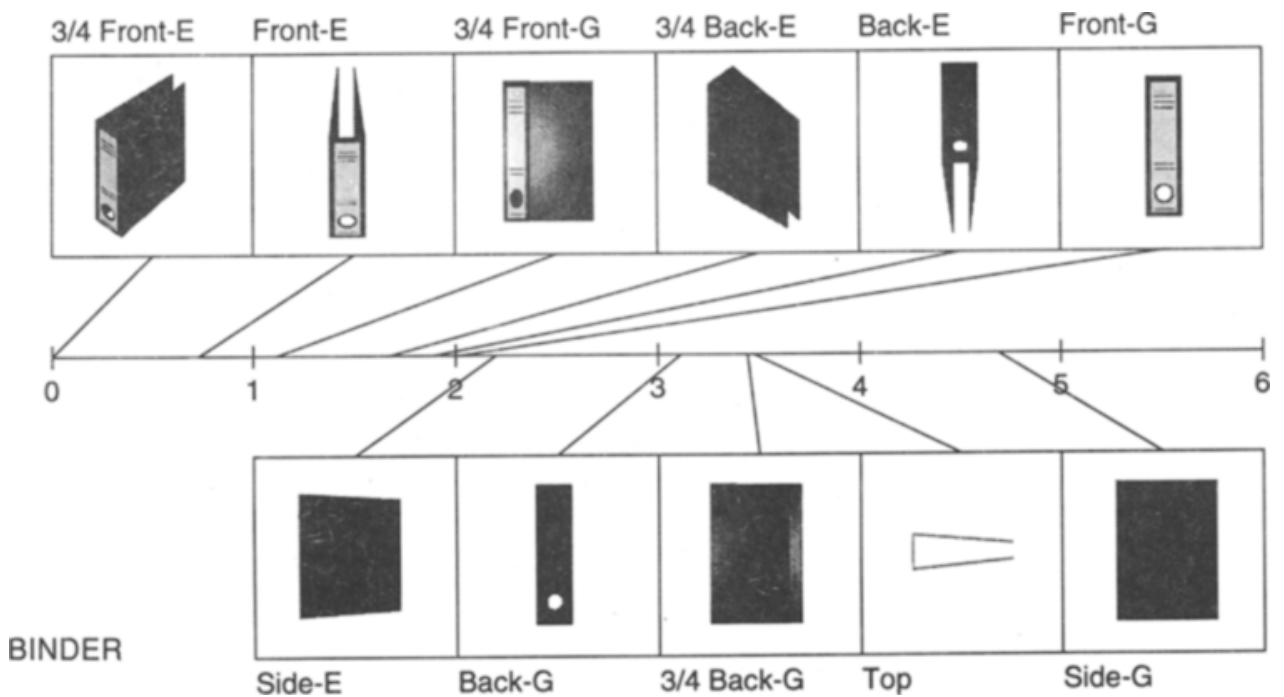

\section{Cluster 7}

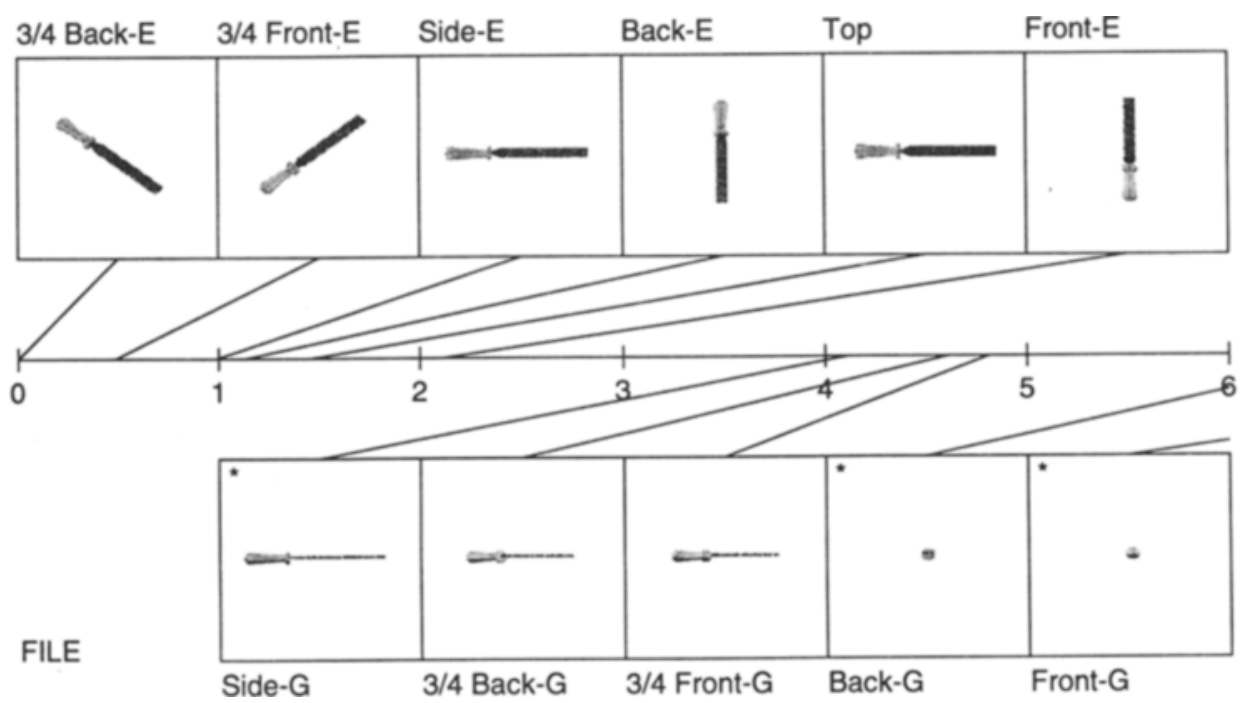




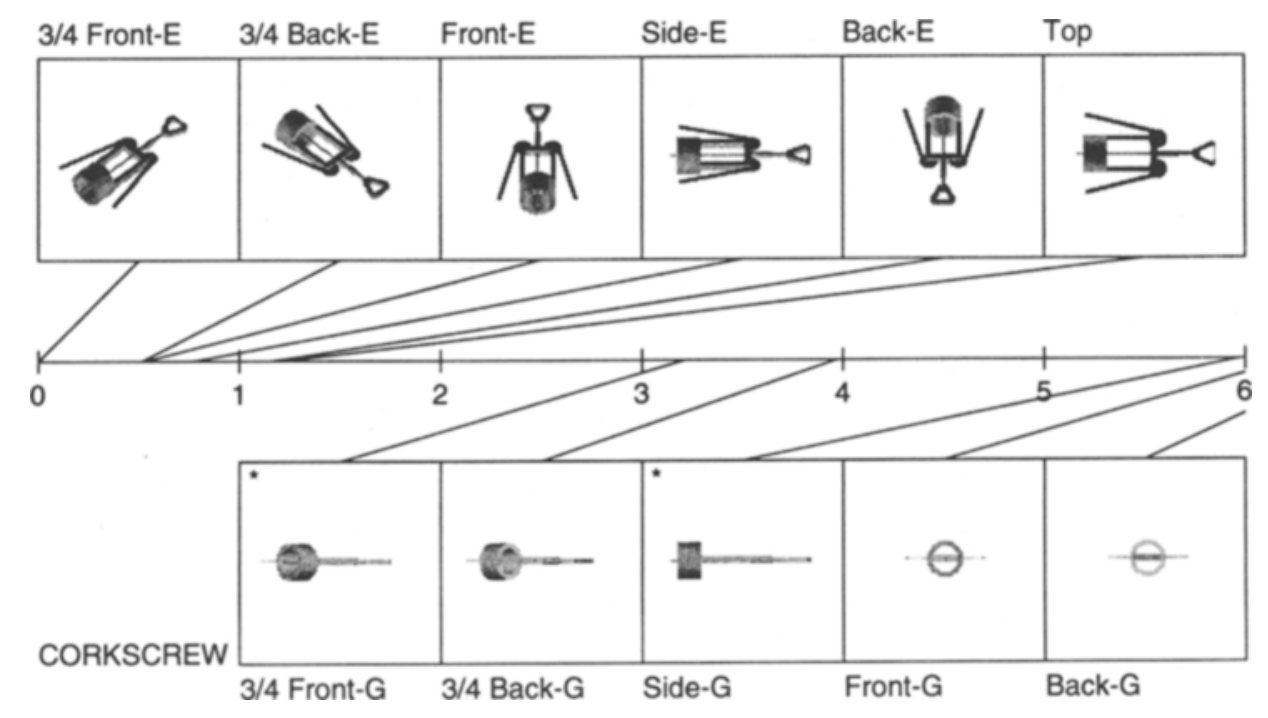

Cluster 8

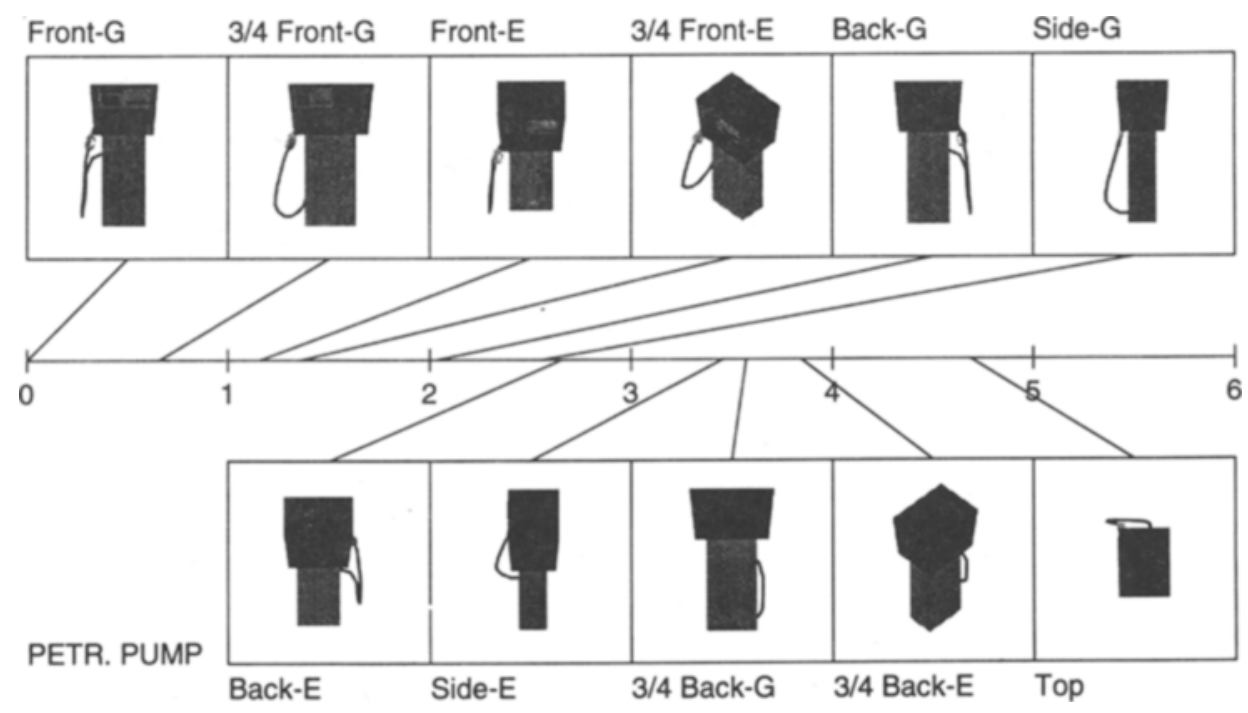


APPENDIX A (Continued)

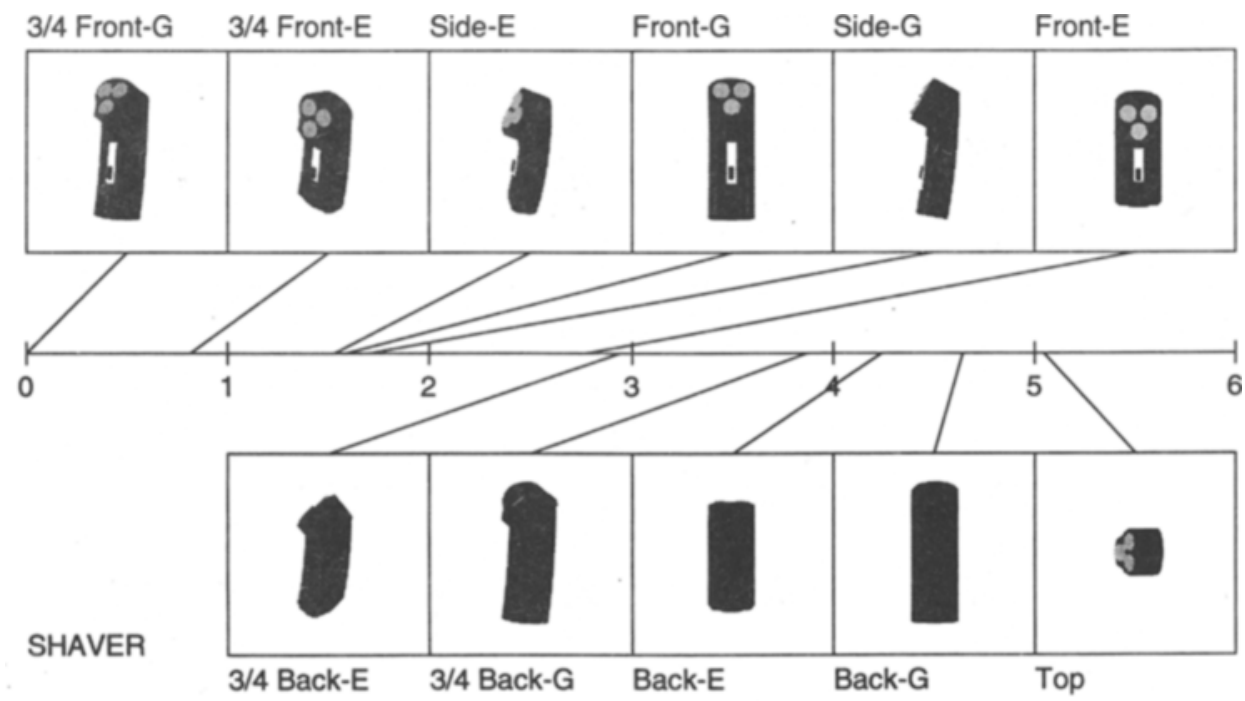

9-VIEW OBJECTS

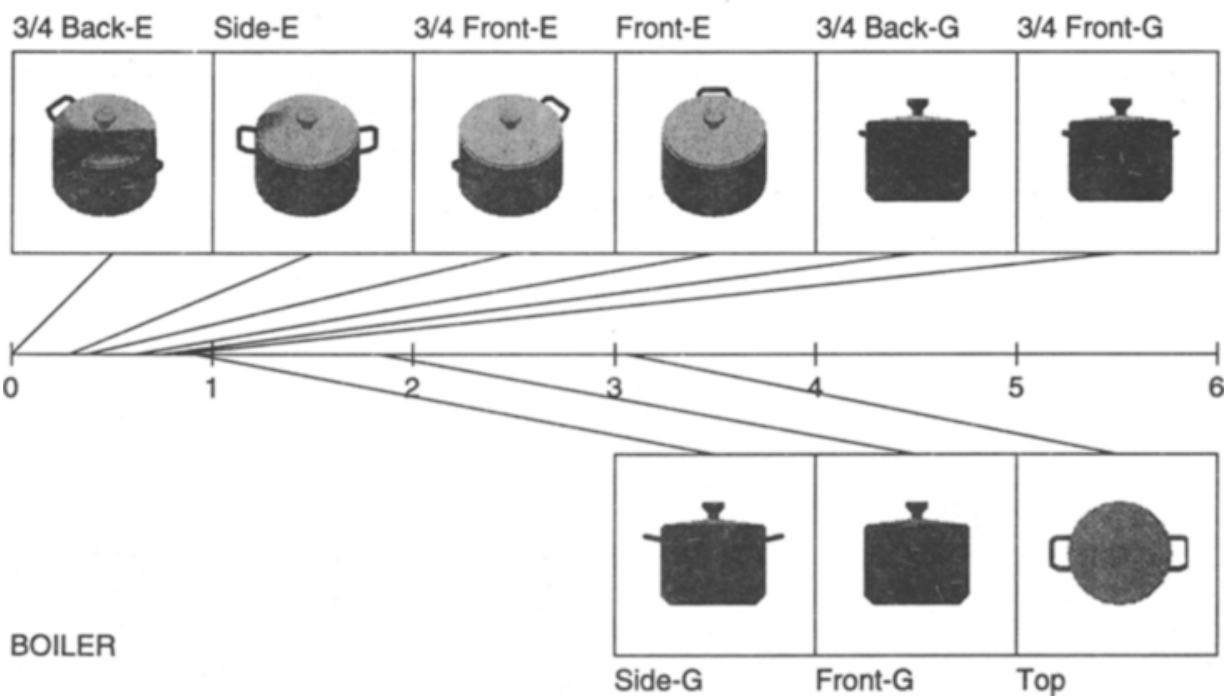



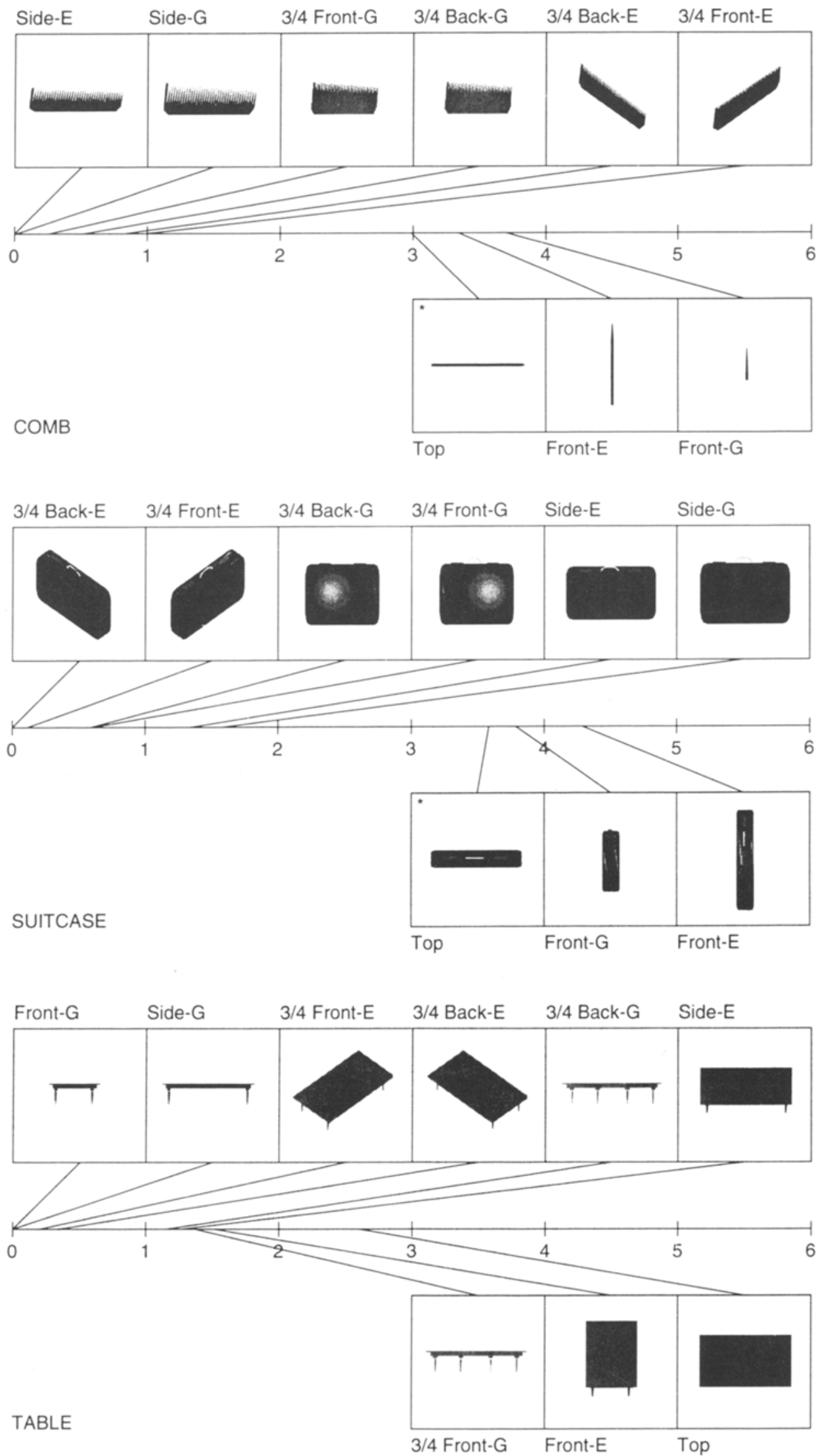
APPENDIX A (Continued)
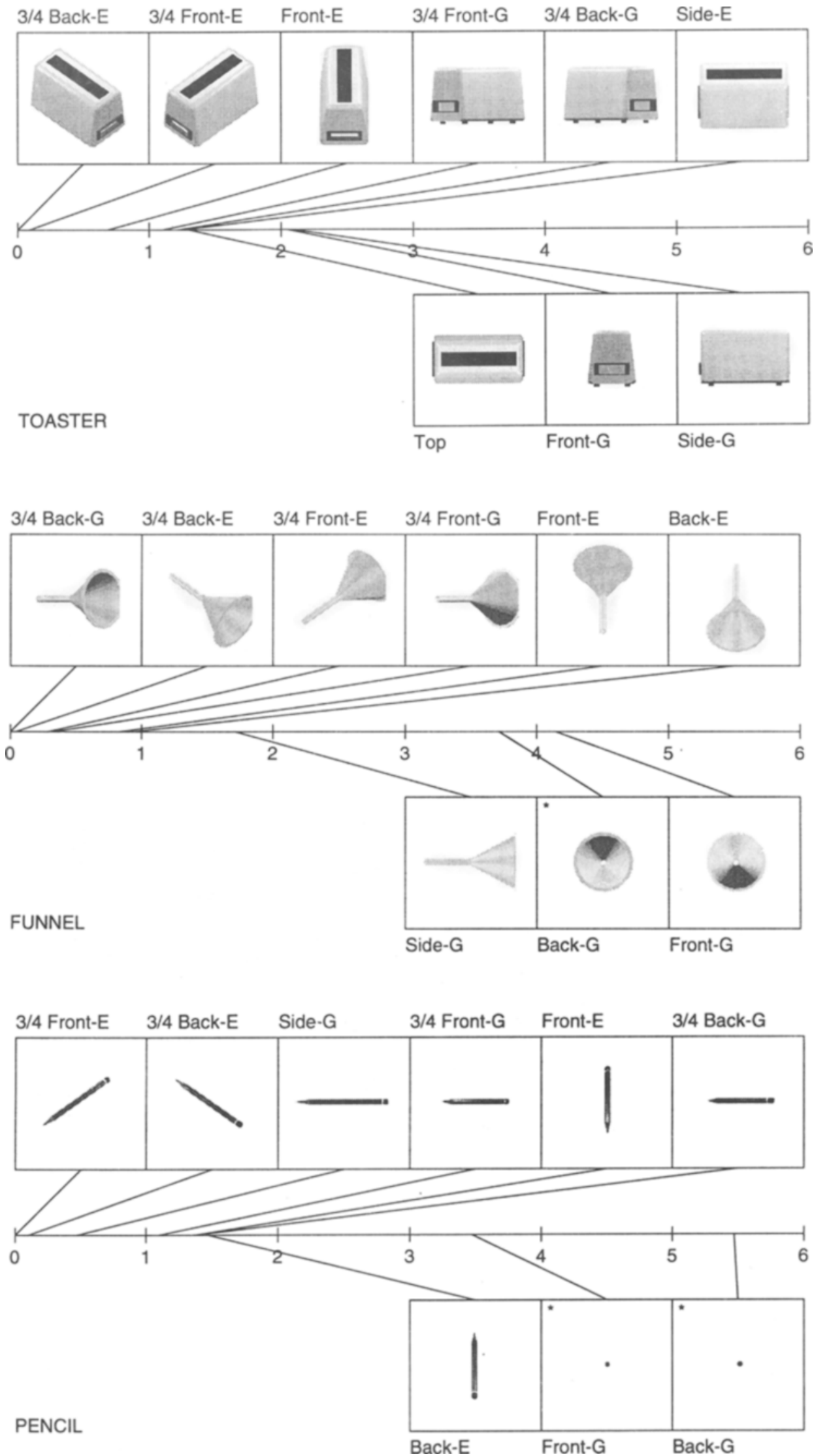


\section{APPENDIX A (Continued)}

7-VIEW OBJECT

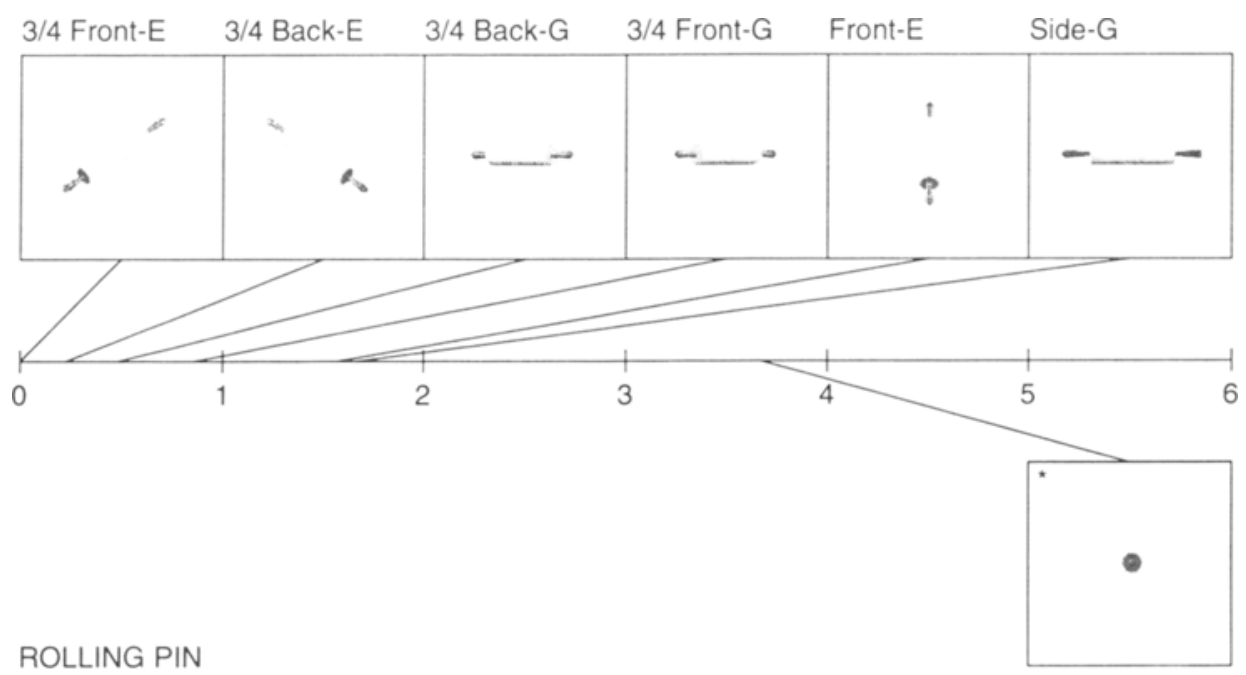

Front-G

\section{5-VIEW OBJECT}

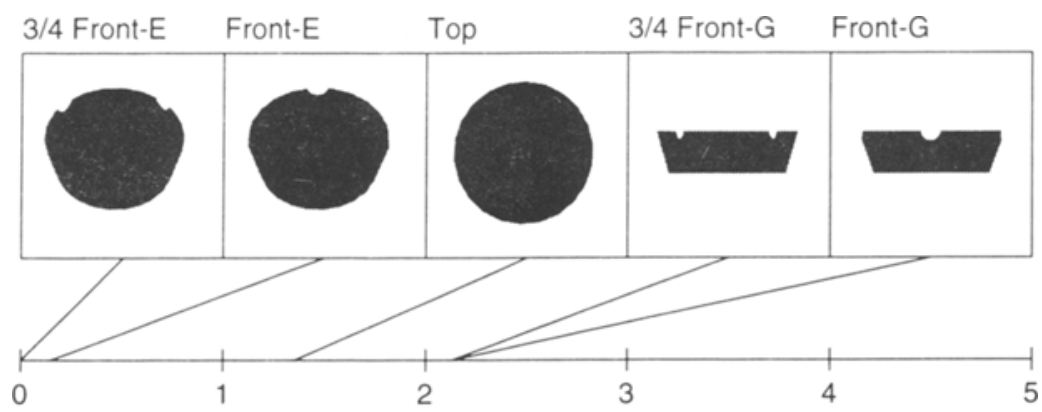

ASHTRAY 


\section{3-VIEW OBJECTS}

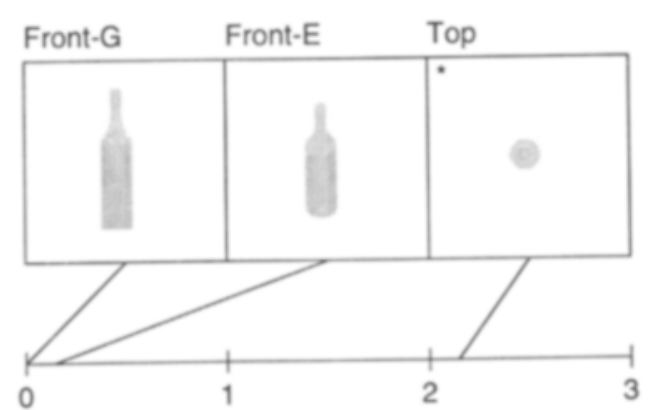

BOTTLE

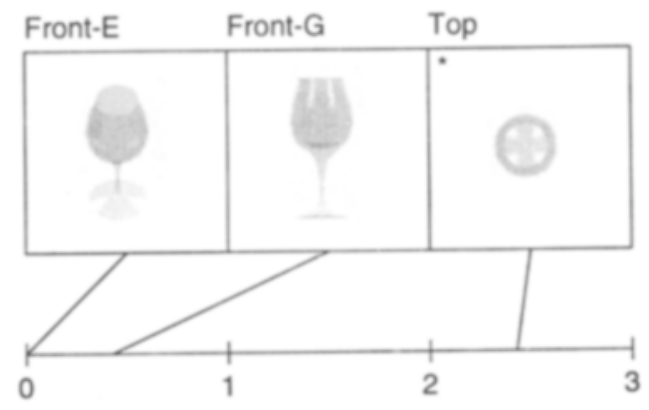

GLASS

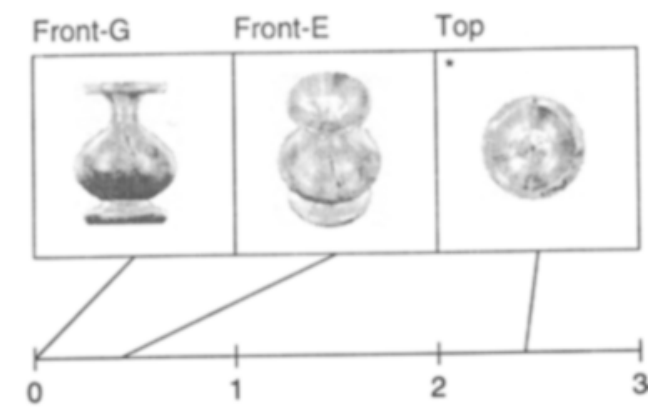

VASE

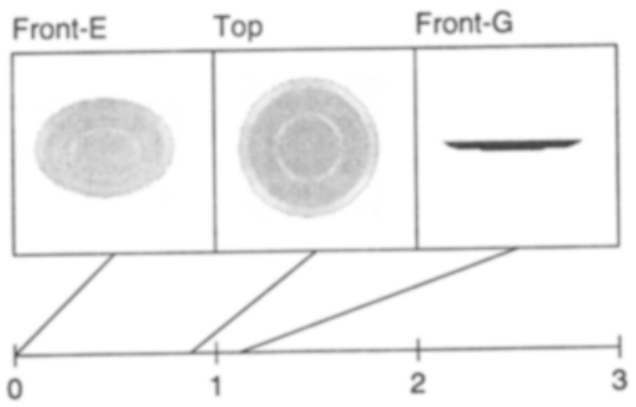

SAUCER 
APPENDIX B

Median Number of Triads, Kendall's (1962) Coefficient of Agreement ( $u$ ), $\chi^{2}$ Test of $u$, Mosteller's (1951) $\chi^{2}$ Test of the Goodness of Fit, and Gulliksen and Tukey's (1958) Coefficient of Reliability for Each Object

\begin{tabular}{|c|c|c|c|c|c|}
\hline & Media & & & Mostell & oefficien \\
\hline Object & Triads & Kendall's $u$ & $\chi_{1 z}^{2}$ & Test & \\
\hline & & 11-View & Objects & & \\
\hline Cluster I & & & & & \\
\hline roller skate & 4 & 0.570 & 402.53 & $61.34^{*}$ & 88.37 \\
\hline piano & 4 & 0.438 & 328.25 & $95.54_{+}^{+}$ & 76.90 \\
\hline stapler & 4 & 0.507 & 367.10 & $84.14_{+}^{+}$ & 82.17 \\
\hline skateboard & 6 & 0.473 & 347.67 & $92.76_{t}^{+}$ & 78.39 \\
\hline tricycle & 6 & 0.539 & 385.39 & $67.68^{*}$ & 86.11 \\
\hline razor blade & 2 & 0.558 & 395.67 & 40.94 & 92.30 \\
\hline fighter jet & 4 & 0.230 & 210.53 & 47.51 & 76.47 \\
\hline shoe & 3 & 0.511 & 369.39 & 53.97 & 88.58 \\
\hline pitchfork & 2 & 0.620 & 431.10 & $121.35_{t}^{*}$ & 80.48 \\
\hline pan & 6 & 0.487 & 355.67 & $107.44+$ & 75.33 \\
\hline Cluster 2 & & & & & \\
\hline ventilator & 6 & 0.394 & 303.10 & $79.08 t$ & 78.87 \\
\hline extinguistier & 7 & 0.406 & 309.96 & 50.68 & 86.05 \\
\hline globe & 12 & 0.348 & 276.82 & $72.20 \dagger$ & 79.07 \\
\hline concrete mixer & r 4 & 0.548 & 389.96 & $98.00_{+}^{+}$ & 80.40 \\
\hline desk lamp & 4 & 0.618 & 429.96 & $123.62_{t}^{+}$ & 79.21 \\
\hline perforator & 2 & 0.495 & 360.24 & $75.49 \dagger$ & 82.18 \\
\hline call box & 5 & 0.346 & 275.67 & 31.99 & 90.37 \\
\hline watch & 4 & 0.564 & 399.10 & 129.53 & 76.44 \\
\hline oil lamp & 3 & 0.628 & 435.67 & $83.85_{\ddagger}^{+}$ & 86.36 \\
\hline Cluster 3 & & & & & \\
\hline $\operatorname{cow}$ & 3 & 0.576 & 405.96 & $63.69^{*}$ & 87.82 \\
\hline helicopter & 2 & 0.630 & 436.82 & 60.45 & 90.19 \\
\hline car & 3 & 0.622 & 432.24 & $118.92_{+}^{+}$ & 80.60 \\
\hline truck & 1 & 0.655 & 450.53 & 55.91 & 90.97 \\
\hline train & 2 & 0.572 & 403.67 & $82.45_{t}^{+}$ & 84.19 \\
\hline roller & 4 & 0.586 & 411.67 & $83.76_{*}^{*}$ & 84.06 \\
\hline chicken & 1 & 0.663 & 455.10 & 51.91 & 91.54 \\
\hline duck & 3 & 0.576 & 405.96 & 51.86 & 90.67 \\
\hline teapot & 4 & 0.647 & 445.96 & 53.34 & 90.77 \\
\hline fork-lift & 2 & 0.604 & 421.96 & 28.76 & 95.10 \\
\hline bench & 1 & 0.636 & 440.24 & 37.12 & 93.56 \\
\hline Cluster 4 & & & & & \\
\hline scissors & 0 & 0.624 & 433.39 & 26.56 & 95.85 \\
\hline spanner & 1 & 0.642 & 443.67 & 17.11 & 97.25 \\
\hline spade & 2 & 0.644 & 444.82 & $72.49 \dagger$ & 87.69 \\
\hline tin opener & 0) & 0.644 & 444.82 & 46.02 & 92.08 \\
\hline racket & 1 & 0.689 & 469.96 & $128.42_{t}^{+}$ & 80.31 \\
\hline rubber dinghy & 0 & 0.707 & 480.24 & $29.19^{\circ}$ & 95.52 \\
\hline hair brush & 3 & 0.665 & 456.24 & $107.07+$ & 83.05 \\
\hline fork & 1 & 0.675 & 461.96 & 61.04 & 90.04 \\
\hline serewdriver & 4 & 0.600 & 419.67 & 36.08 & 93.75 \\
\hline Cluster 5 & & & & & \\
\hline ax & 2 & 0.646 & 443.67 & 34.84 & 94.55 \\
\hline bicycle & 4 & 0.620 & 431.10 & 20.72 & 96.56 \\
\hline
\end{tabular}

\section{APPENDIX B (Continued)}

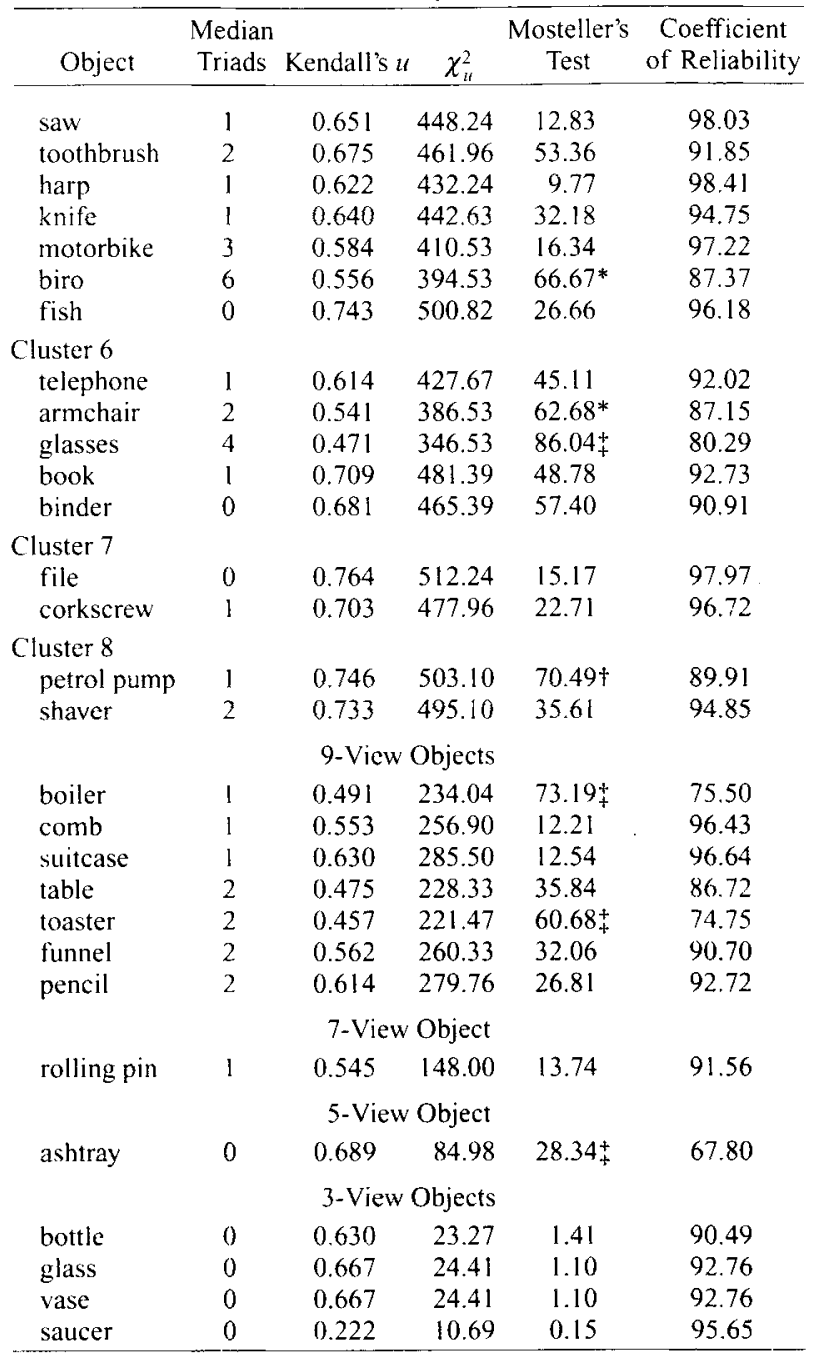

Note-For $\chi^{2}, d f=81$ for 11 -view objects, $d f=53$ for 9-view objects, $d f=31$ for the 7 -view object, $d f=15$ for the 5 -view object, and $d f=4$ for 3-view objects: $p<.001$, for all objects, except for the saucer $(p<.05)$. For Mosteller's test, $d f=45$ for 11 -view objects, $d f=28$ for 9 -view objects, $d f=15$ for the 7 -view object, $d f=6$ for the 5 -view object, and $d f=$ I for 3-view objects. ${ }^{*} p<.05$. $\dagger p<.01 . \neq p<.001$.
(Manuscript received December 1, 1994; revision accepted for publication March 13, 1995.) 